Pacific Journal of Mathematics

APPROXIMATION PROPERTIES FOR SOM 


\section{APPROXIMATION PROPERTIES FOR SOME NON-NOETHERIAN LOCAL RINGS}

\section{H. SCHOUTENS}

In this paper we study Artin approximation in power series rings in several variables over complete rank-one valuation rings. In particular we prove that the completion of the algebraic elements has the approximation property over the ring of algebraic power series.

Moreover, for an important class of complete rank-one valuation rings, e.g. the ring of complex $p$-adic integers, we prove that the ring of algebraic power series is equal to the henselisation of the polynomial ring and that each algebraic power series has coefficients lying in a finitely generated $R$-algebra, where $R$ is discrete valuation rings.

1.1. Let $R \subset \bar{R}$ be a pair of rings (always commutative, with unity). We'll consider topologies on $\bar{R}$ which stem from a filtration of ideals $a_{0} \supset a_{1} \supset a_{2} \supset \cdots$ which tends to zero, i.e. $\bigcap_{n=0}^{\infty} a_{n}=0$. Two examples we will use are given by

(1) an ideal $a$ of $\bar{R}$ with $\bigcap_{n=0}^{\infty} a^{n}=0$ and where $a_{n}=a^{n}$; thus we get the a-adic topology,

(2) a rank-one valuation on $\bar{R}$ (i.e. a valuation with value group in the positive real numbers) and $a_{n}=\{x \in \bar{R} \mid v(x) \geq n\}$.

Definition. $\bar{R} / R$ has $A$. $P$. (( Artin)-approximation property) when the following holds: For every system of polynomial equations $f=0$ over $R$, i.e. $f=\left(f_{1}, \ldots, f_{q}\right)$ with $f_{i} \in R\left[Y_{1}, \ldots, Y_{N}\right]$ which has a solution $\bar{y}$ in $\bar{R}^{N}$, we can find for each $n$ in $\mathbf{N}$ a solution $y$ in $R^{N}$ such that $\bar{y} \equiv y$ $\bmod a_{n}$.

REMARK. Often, one can express congruence conditions such as " $\bar{y} \equiv y \bmod a_{n}$ " appearing in the definition, by polynomial conditions. More explicitly, let $I$ be a finitely generated ideal of $R$, such that $R$ is dense in $\bar{R}$ with respect to the $I \bar{R}$-adic topology. Let $f \in R[Y]^{q}, Y=$ $\left(Y_{1}, \ldots, Y_{N}\right)$ and $\bar{y} \in \bar{R}^{N}$ s. t. $f(\bar{y})=0$. We look for a solution $y \in R^{N}$ such that $y \equiv \bar{y} \bmod I^{m}$, for a chosen $m \in \mathbf{N}$. Let $I^{m}=\left(q_{1}, \ldots, q_{s}\right)$ with $q_{i} \in R$, and since $R \subset \bar{R}$ is dense, we can find $\dot{y} \in R^{N}, \bar{\alpha}_{1}, \ldots, \bar{\alpha}_{s} \in \bar{R}^{N}$ s.t. $\bar{y}=\dot{y}+\bar{\alpha}_{1} q_{1}+\cdots+\bar{\alpha}_{s} q_{s}$. 
Consider the polynomial system over $R$ given by

$$
\begin{aligned}
& P(Y)=0, \\
& Y-\left(\dot{y}+q_{1} Z_{1}+\cdots+q_{s} Z_{s}\right)=0
\end{aligned}
$$

in the variables $Y, Z_{1}=\left(Z_{11}, \ldots, Z_{1 N}\right), \ldots, Z_{s}=\left(Z_{s 1}, \ldots, Z_{s N}\right)$ which has a solution $\left(\bar{y}, \bar{\alpha}_{1}, \ldots, \bar{\alpha}_{s}\right) \in \bar{R}^{N(s+1)}$. By A.P. we find a solution $\left(y, \alpha_{1}, \ldots, \alpha_{s}\right) \in R^{N(s+1)}$, hence $y=\dot{y}+\sum_{i=1}^{s} q_{i} \alpha_{i}$, so $y \equiv \dot{y} \bmod I^{m}$, so $y \equiv \bar{y} \bmod I^{m}$ and $f(y)=0$.

1.2. A particularly interesting situation for A.P. is when we take for $A$ a local ring, $\bar{R}=A[[X]]$ and $R=A[[X]]^{h}$, where $X=\left(X_{1}, \ldots, X_{n}\right)$ are several variables and " $h$ " denotes the henselisation of $A[X]_{(X)}$ at the maximal ideal. So, one can ask whether the following holds

$$
A[[X]] / A[[X]]^{h} \text { has A.P. }
$$

Artin has proven (1) for $A$ a field or an excellent discrete valuation ring (D.V.R.) [Ar].

But for $A$ a non-Noetherian excellent (or even complete) rank-one valuation ring the answer is still not known. A typical complete rank-one valuation ring we will be concerned with is $\mathcal{O}_{\mathbf{C}_{p}}$ the ring of complex integers, i.e. the integral elements of $\mathbf{C}_{p}$, where $\mathbf{C}_{p}$ is the $p$-adic closure of the algebraic closure of the $p$-adic numbers $\mathbf{Q}_{p}$ ( $p$ being a rational prime). The problem in proving (1) for the non-Noetherian situation is the absence of a good desingularisation theorem. A consequence of (1) for $A$ a domain is

$$
A[[X]]^{h}=A[[X]]^{\text {alg }}
$$

where $A[[X]]^{\text {alg }}$ is the ring of algebraic power series, i.e. the power series which are algebraic over Frac $A[X]$. Property (2) is well-known for $A$ an excellent domain, but for non-Noetherian rings again this is not known in general.

We will be able to prove (2) for a special kind of complete rank-one valuation rings, including the case of $\mathcal{O}_{C_{p}}$, see 4.4.2. At the same time, we will prove for this kind of rings a generalisation in more variables of a result of Christol [Chr; Prop. 7.2.]. (See Theorem 4.2.2.) As a result we obtain for instance

every algebraic power series of $\mathcal{O}_{\mathbf{C}_{p}}[[X]]$ has coefficients lying in a finitely generated $\mathbf{Z}_{p}$-algebra,

where $\mathbf{Z}_{p}$ is the ring of $p$-adic integers in $\mathbf{Q}_{p}$ and $X=\left(X_{1}, \ldots, X_{n}\right)$ are several variables, see Thm. 4.4.2. 
1.3. Another interesting case of A.P. arises when one takes a complete rank-one valuation ring $A$ and for $R=A[[X]]^{\text {alg }}$, the ring of algebraic power series in $X=\left(X_{1}, \ldots, X_{n}\right)$ and for $\bar{R}$ the completion of $R$ in $A[[X]]$ with respect to the valuation. We will denote this by $\bar{R}=A[[\hat{X}]]^{\text {alg }}$. We will prove:

$$
A[[\hat{X}]]^{\mathrm{alg}} / A[[X]]^{\mathrm{alg}} \text { has A.P. in }(X) \text {-topology. }
$$

Robba has proven the same statement in characteristic zero only, but with respect to the Gaussnorm [Ro]. (When $v$ is the valuation on $A$, then we mean by the Gaussnorm on $A[[X]]: v\left(\sum_{i \in \mathbf{N}^{n}} a_{i} X^{i}\right)=\inf \left\{v\left(a_{i}\right) \mid i \in\right.$ $\left.\mathbf{N}^{n}\right\}, X=\left(X_{1}, \ldots, X_{n}\right)$.) But by the remark in 1.1 his statement is actually equivalent with ours, by taking for $I=(\pi) A[[X]]^{\text {alg }}$, with $\pi \in A$ and $v(\pi)>0$.

1.4. Because we need several A.P. theorems of the same type $(\bar{R}$ is the completion of $R$ with respect to a rank-one valuation, e.g. (4)), we prove A.P. in a more general situation for systems of subrings of $A[[X]]$ and their completions, called pseudo-power series; this is done in Theorem 2.4.1.

1.5. In the last paragraph we have brought together all Weierstrass Preparation Theorems (W.P.T.) we will need. For lack of good reference, we have estimated it opportune to write out these theorems in a rather detailed way.

1.6. We would like to thank Dr. J. Denef for presenting these problems and for his suggestion to use 4.3.4 in order to obtain result (2).

\section{Approximation property.}

2.1.1. Definition. Let $A$ be an arbitrary domain with fraction field $F$. We'll say that $A$ is completely integrally closed (C.I.C.) if for $x \in F$ with $d \in A, d \neq 0$ so that $\forall n \in \mathbf{N}: d x^{n} \in A$ implies that $x \in A$ (also called completely normal). In [Ma; (17.B)] one proves that when $A$ is C.I.C., so are $A[X]$ and $A[[X]]$.

2.1.2. TheOREM (Fatou-property) When $A$ is C.I.C., T one variable and $S=1+T \cdot A[[T]]$ then $A[[T]] \cap \operatorname{Frac}(A[T])=S^{-1} A[T]$. 
Proof. Cf. [Ch; §3].

2.2.1. Let $A$ be an arbitrary ring, $a$ an ideal of $A$, with $a \subset \operatorname{rad} A$ (Jacobson radical of $A$ ).

We call $(A, a)$ a Henselian pair, if

$$
\forall f=\left(f_{1}, \ldots, f_{N}\right), \quad f_{l} \in A[X], \quad X=\left(X_{1}, \ldots, X_{N}\right)
$$

and $x \in A^{N}, x=\left(x_{1}, \ldots, x_{N}\right)$ such that

$$
f(x) \equiv 0 \quad \bmod a
$$

and $\left.\operatorname{det}\left(\partial f_{i} / \partial X_{J}\right)\right|_{x}$ is invertible, then

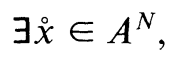

such that

$$
f(\stackrel{\circ}{x})=0 \text { and } x \equiv \stackrel{\circ}{x} \quad \bmod a .
$$

This is just a generalisation of Henselian local rings and analogous properties hold. See [Ra]. Especially we'll use:

2.2.2. LeMma (Tougéron). Let $(A, a)$ be a Henselian pair, $f=$ $\left(f_{1}, \ldots, f_{h}\right), f_{l} \in A[Y], Y=\left(Y_{1}, \ldots, Y_{N}\right)$. Let $J=\left(\partial f_{i} / \partial X_{j}\right)$ be the Jacobian and $\delta$ a $h \times h$-minor of $J$. Suppose there is an $x \in A^{N}$ such that $f(x) \equiv 0 \bmod \delta(x)^{2} a$. Then $\exists \dot{x} \in A^{N}$ such that

$$
f(\stackrel{\circ}{x})=0 \text { and } x \equiv \stackrel{\circ}{x} \bmod \delta(x) a .
$$

Proof. See [Ar; II §4.1].

2.3.1. Let $A$ be a ring, then we shall mean by a pseudo-power series-system (PP-system) a system of rings $\stackrel{\circ}{W}_{n}, n=0,1,2, \ldots$, such that

(1) $\stackrel{\circ}{W}_{0}=A$,

(2) $\stackrel{\circ}{W}_{n-1}\left[X_{n}\right] \subset \stackrel{\circ}{W}_{n} \subset \stackrel{\circ}{W}_{n-1}\left[\left[X_{n}\right]\right]$ for $n=1,2, \ldots$,

(3) $\left(X_{1}, \ldots, X_{n}\right) \subset \operatorname{rad} \stackrel{\circ}{W}_{n}$ for $n=1,2, \ldots$,

(4) If $\varphi_{1}, \ldots, \varphi_{n} \in\left(X_{1}, \ldots, X_{n}\right) A\left[X_{1}, \ldots, X_{n}\right]$ and $\lambda\left(X_{1}, \ldots, X_{n}\right) \in$ $\stackrel{\circ}{W}_{n}$ then $\lambda\left(\varphi_{1}, \ldots, \varphi_{n}\right) \in \stackrel{\circ}{W}_{n}$. Note that then $A\left[X_{1}, \ldots, X_{n}\right] \subset \dot{\circ}_{n} \subset$ $A\left[\left[X_{1}, \ldots, X_{n}\right]\right]$. Clearly the powers series form a PP-system.

2.3.2. In this paragraph we'll work from now on with a valuation ring 0 which is complete with respect to its valuation ord. Let $\mathscr{M}$ be the maximal ideal and $K=$ Frac $\mathcal{O}$ the fraction field. We can extend ord to a 
valuation on $\mathcal{O}[[X]]$ also written ord by setting

$$
\operatorname{ord}\left(\sum a_{i} X^{i}\right)=\inf \left\{\operatorname{ord}\left(a_{i}\right)\right\}
$$

with $i=\left(i_{1}, \ldots, i_{n}\right), X=\left(X_{1}, \ldots, X_{n}\right)$ and $X^{i}=X_{1}^{i_{1}} \cdots X_{i}^{i_{i}} \cdots X_{n}^{i_{n}}$. We call $f=\sum a_{i} X^{i} \in \mathcal{O}[[X]]$ separable when $\exists i_{0}: \operatorname{ord}(f)=\operatorname{ord}\left(a_{i_{0}}\right)$, i.e. the infimum actually is a minimum. E.g. polynomials are separable. Let $\stackrel{\circ}{W}_{n}$ be a PP-system over $\mathcal{O}$ and denote by $W_{n}$ the completion of $\dot{W}_{n}$ in $\mathcal{O}\left[\left[X_{1}, \ldots, X_{n}\right]\right]$. Then $W_{n}$ also forms a PP-system.

We will call an $f \in \mathcal{O}\left[\left[X_{1}, \ldots, X_{n}\right]\right]$ regular of degree $k$ in $X_{n}$ when $f \equiv X_{n}^{k} u \bmod \left(\mathscr{M}, X^{\prime}\right)$ with $u$ unit in $\mathcal{O}\left[\left[X_{1}, \ldots, X_{n}\right]\right]$ and $X^{\prime}=$ $\left(X_{1}, \ldots, X_{n-1}\right)$ (cf. §5).

By saying that the PP-system $\dot{W}_{n}$ has a Weierstrass preparation theorem (W.P.T.) we shall mean that for each $f \in \mathscr{W}_{n}$ which is regular in $X_{n}$ of degree $k$ and

$$
\forall g \in \stackrel{\circ}{W}_{n}: \exists \text { unique } q \in \stackrel{\circ}{W}_{n}, \quad r \in \stackrel{\circ}{W}_{n-1}\left[X_{n}\right]
$$

such that

$$
g=q \cdot f+r \quad \text { with } \quad \operatorname{deg}_{X_{n}}(r)<k .
$$

2.4.1. Theorem (Approximation property, A.P.). Set $X=$ $\left(X_{1}, \ldots, X_{n}\right)$. Let $\stackrel{\circ}{n}_{n}$ be a PP-system over $\mathcal{O}, W_{n}$ its completion. Suppose that the following conditions hold $\forall n$ :

(a) $\forall \pi \in \mathscr{M}: \pi \mathcal{O}[[X]] \cap \stackrel{\circ}{W}_{n}=\pi \stackrel{\circ}{W}_{n}$,

(b) $W_{n}$ has a W.P.T.,

(c) $\forall f \in W_{n}: f$ is separable,

(d) $W_{n}$ is C.I.C.,

(e) $\left(\stackrel{\circ}{W}_{n}, X\right)$ is a Henselian pair,

(f) Frac $W_{n}$ is separable over Frac $\stackrel{\circ}{W}_{n}$.

Then $W_{n} / \stackrel{\circ}{W}_{n}$ has the A.P. with respect to polynomial equations, i.e. with $Y=\left(Y_{1}, \ldots, Y_{N}\right)$

$$
\begin{gathered}
\forall f=\left(f_{1}, \ldots, f_{q}\right) \quad f_{i} \in \mathcal{O}[X][Y] \\
\forall c \in \mathbf{N}_{0} \text { and } \bar{y}=\left(\bar{y}_{1}, \ldots, \bar{y}_{N}\right), \quad \bar{y}_{i} \in W_{n}
\end{gathered}
$$

so that $f(X, \bar{y})=0$.

Then $\exists \dot{y}=\left(\dot{y}_{1}, \ldots, \dot{y}_{N}\right), \dot{y}_{i} \in \stackrel{\circ}{W}_{n}$ so that

$$
f(X, \stackrel{\circ}{y})=0 \text { and } \bar{y} \equiv \stackrel{\circ}{\bmod }(X)^{c} .
$$


Proof. We'll write $W$ for $W_{n}$ and $\stackrel{\circ}{W}$ for $\stackrel{\circ}{W}_{n}$. We will do induction on $n, n=0$ being trivial.

Let $\psi: \stackrel{W}{W}[Y] \rightarrow W: \alpha(Y) \mapsto \alpha(\bar{y})$ so $\not=\operatorname{ker} \psi$ is a prime ideal and we have

$$
\stackrel{\circ}{L}=\operatorname{Frac} \stackrel{\circ}{W} \subset E=\operatorname{Frac}\left(\frac{\stackrel{\circ}{W}[Y]}{\not h}\right) \subset L=\operatorname{Frac} W .
$$

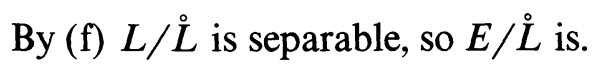

Call $m=\operatorname{trdeg}_{L} E$ (transcendence degree), and after a transformation we may suppose that

$$
E \text { is algebraic separable over } \stackrel{\circ}{L}\left(\bar{Y}_{1}, \ldots, \bar{Y}_{m}\right)
$$

where "_" denotes residues $\bmod \not p$.

So, there exist $\varphi_{i}(T) \in \stackrel{\circ}{W}\left[Y_{1}, \ldots, Y_{m}\right][T]$

$T$ one variable, $i=m+1, \ldots, N$, minimal polynomials of the $\bar{Y}_{i}$ so that

$$
h_{i}=\varphi_{i}\left(Y_{i}\right) \in \not h
$$

and

$$
\frac{\partial h_{i}}{\partial Y_{i}}=\varphi_{i}^{\prime}\left(Y_{i}\right) \notin \not h .
$$

Let $\delta=\prod_{i=m+1}^{N}\left(\partial h_{i} / \partial Y_{i}\right) \notin \not p$, so $\delta$ is a $(N-m) \times(N-m)$ minor of the Jacobian $\left(\partial h_{i} / \partial Y_{i}\right)$.

Assertion 1. $\exists \theta \in \stackrel{\circ}{W}[Y], \theta \notin \not 2$ so that

$$
\forall i=1, \ldots, q: \theta f_{\imath} \in\left(h_{m+1}, \ldots, h_{N}\right) .
$$

We claim that it is sufficient to prove the theorem for the system of equations $h_{m+1}, \ldots, h_{N}$. For let $\stackrel{\circ}{y} \in \mathscr{W}^{N}$ be a solution with $\bar{y} \equiv$ $\dot{y} \bmod (X)^{c}$ and $h_{i}(\dot{y})=0$. Since $\theta \notin \not$, we have $\theta(\bar{y}) \neq 0$, choose $c$ big enough so that $\theta(\bar{y}) \notin(X)^{c}$, therefore $\theta(\dot{y}) \notin(X)^{c}$, so $\theta(\dot{y}) \neq 0$, but $\theta f_{i} \in\left(h_{m+1}, \ldots, h_{N}\right)$ by ass. 1 so $\left(\theta f_{i}\right)(\dot{y})=0 \Rightarrow f_{i}(\dot{y})=0$.

This proves the claim, and we therefore may suppose that we have a polynomial system of equations $F_{1}, \ldots, F_{h}$ with $\delta$ a (maximal) $h \times h$ minor out of the Jacobian

$$
J=\left(\frac{\partial F_{i}}{\partial Y_{J}}\right)_{l=1, \ldots, h / J=1, \ldots, N} \quad \text { with } \delta(\bar{y}) \neq 0, \quad \text { and } \quad h=N-m .
$$


Suppose

$$
\delta=\operatorname{det}\left(\frac{\partial F_{i}}{\partial Y_{j}}\right)_{i, j=1, \ldots, h} \text { and let } \varepsilon=\operatorname{ord} \delta(\bar{y}) .
$$

$\exists h \times h$ matrix $Q$ over $\stackrel{\circ}{W}[Y]$ with $\operatorname{det} Q=\delta^{h-1}$ so that

$$
Q \cdot J=\left(\delta E_{h} K\right)
$$

where $E_{h}$ is the identity-matrix and $K$ an $h \times m$ matrix. Since $W$ is the completion of $\stackrel{\circ}{W}$

$$
\begin{gathered}
\exists \gamma, \pi \in \mathcal{O}, \quad \alpha \in \stackrel{\circ}{W}^{N}, \quad \bar{z} \in W^{N} \quad \text { so that } \\
\bar{y}=\alpha+\pi^{2} \gamma \bar{z} \quad \text { with ord } \pi=\varepsilon \text { and ord } \gamma>0 .
\end{gathered}
$$

Set $Z=\left(Z_{1}, \ldots, Z_{N}\right)$ and define, in vector-notation,

$$
\begin{aligned}
G(Z)= & \frac{Q(\alpha)}{\pi^{2}} \\
& \cdot F\left(\alpha_{1}+\pi Z_{1}, \ldots, \alpha_{h}+\pi Z_{h}, \alpha_{h+1}+\pi^{2} Z_{h+1}, \ldots, \alpha_{N}+\pi^{2} Z_{N}\right) \\
= & \frac{Q(\alpha)}{\pi^{2}} \cdot F(\alpha)+\frac{Q(\alpha) \cdot J(\alpha)}{\pi^{2}} \\
& \cdot\left(\pi Z_{1}, \ldots, \pi Z_{h}, \pi^{2} Z_{h+1}, \ldots, \pi^{2} Z_{N}\right)+\mu(Z)
\end{aligned}
$$

with $\mu(Z)$ in $(Z)^{2} \stackrel{\circ}{W}[Z]$.

Since ord $(\alpha-\bar{y})>2 \varepsilon$ we have

$$
\begin{gathered}
0=F(\bar{y}) \equiv F(\alpha) \bmod \pi^{2}, \quad \text { so ord } F(\alpha) \geq 2 \varepsilon \\
\text { ord } \delta(\bar{y})=\operatorname{ord} \delta(\alpha)=\varepsilon .
\end{gathered}
$$

So by Property (a), $F(\alpha) / \pi^{2}, \delta(\alpha) / \pi \in \stackrel{\circ}{W}$ and

$$
\operatorname{ord}\left(\frac{\delta(\alpha)}{\pi}\right)=0
$$

So by (1) we get

$$
\begin{aligned}
G(Z)= & Q(\alpha) \cdot \frac{F(\alpha)}{\pi^{2}}+\frac{\delta(\alpha)}{\pi}\left(Z_{1}, \ldots, Z_{h}\right) \\
& +K(\alpha) \cdot\left(Z_{h+1}, \ldots, Z_{N}\right)+\mu(Z)
\end{aligned}
$$

lies in $\stackrel{\circ}{W}[Z]$.

Let $\bar{w}=\left(\pi \gamma \bar{z}_{1}, \ldots, \pi \gamma \bar{z}_{h}, \gamma \bar{z}_{h+1}, \ldots, \gamma \bar{z}_{N}\right)$, so ord $\bar{w}>0$ and by (2)

$$
G(\bar{w})=\frac{Q(\alpha)}{\pi^{2}} F\left(\alpha+\pi^{2} \gamma \bar{z}\right)=0 .
$$


Suppose we can solve the theorem for the system $G=0$ with solution $\bar{w}$ in $W$, and a $h \times h$ minor of the Jacobian in $\bar{w}$ of $G$ which is equal to $(\delta(\alpha) / \pi)^{h} \bmod \mathscr{M}$ i.e. of valuation equal to zero by (3). Then we can also solve it for our original system $F$; indeed, let $\stackrel{\circ}{w}$ be in $\stackrel{\circ}{W}$ so that $G(\stackrel{\circ}{w})=0$ and $\bar{w} \equiv \stackrel{\circ}{w} \bmod (X)^{c}$, then, if we put $\dot{y}=\left(\alpha_{1}+\pi \stackrel{\circ}{w}_{1}, \ldots, \alpha_{h}+\pi \stackrel{\circ}{w}_{n}\right.$, $\left.\alpha_{h+1}+\pi^{2} \dot{\circ}_{h+1}, \ldots, \alpha_{N}+\pi^{2} \dot{\circ}_{N}\right)$,

$$
0=G(\stackrel{\circ}{w})=\frac{Q(\alpha)}{\pi^{2}} \cdot F(\stackrel{\circ}{y} \text {. }
$$

And since $\operatorname{det} Q(\alpha)=\delta(\alpha)^{h-1} \neq 0$, we get $F(\dot{y})=0$ and clearly is $\bar{y} \equiv$ $\stackrel{\circ}{y} \bmod (X)^{c}$ and $\dot{y}$ lies in $\stackrel{\circ}{W}$.

So we may suppose that $\varepsilon=\operatorname{ord} \delta(\bar{y})=0$ from the start. Since $\delta(\bar{y}) \in W$, by property (c) we know then that $\delta(\bar{y})$ is separable and that it is regular in $X_{n}$ after a transformation of the form $X_{j} \mapsto X_{j}+X_{n}^{u_{j}}$, $j=1, \ldots, n-1$ and $X_{n} \mapsto X_{n}$ (see for instance [Z-S; VII §1]) which keeps $W_{n}$ invariant by (4) of PP-systems. Applying the W.P.T. (property (b)) for $g(\bar{y}) X_{n}^{c}=\delta^{2}(\bar{y}) X_{n}^{c}$, which is regular in $X_{n}$ of, say, degree $s$, we get:

$$
\bar{y}=u \cdot g(\bar{y}) X_{n}^{c}+\tilde{y}
$$

with $u \in W_{n}$ and $\tilde{y} \in W_{n-1}\left[X_{n}\right] \subset W_{n}$ so

$$
0=F(\bar{y}) \equiv F(\tilde{y}) \bmod g(\bar{y}) X_{n}^{c}, \quad g(\bar{y}) \equiv g(\tilde{y}) \bmod g(\bar{y}) X_{n}^{c}
$$

so

$$
\exists \lambda \in W_{n}: g(\bar{y})=g(\tilde{y})-\lambda g(\bar{y}) X_{n}^{c} \Rightarrow\left(1+\lambda X_{n}^{c}\right) g(\bar{y})=g(\tilde{y})
$$

and since $(X) \subset \operatorname{rad} W_{n}$ we have that $g(\bar{y})$ and $g(\tilde{y})$ are associated in $W_{n}$. So by (4)

$$
F(\tilde{y}) \equiv 0 \quad \bmod g(\tilde{y}) X_{n}^{c}
$$

so

$$
\exists \tilde{z} \in W_{n}: F(\tilde{y})=\tilde{z} g(\tilde{y}) X_{n}^{c} .
$$

Call $\tilde{W}_{n-1}=W_{n-1}\left[X_{n}\right] \subset W_{n}$, and since $\tilde{y} \in \tilde{W}_{n-1}$ also $F(\tilde{y})$ and $g(\tilde{y}) X_{n}^{c} \in \tilde{W}_{n-1}$.

Let $S=1+X_{n} \cdot \tilde{W}_{n-1}$, we have then by property (d) and Theorem 2.1.2 that, since $W_{n} \subset W_{n-1}\left[\left[X_{n}\right]\right]$

$$
\tilde{z}=F(\tilde{y}) / g(\tilde{y}) X_{n}^{c} \in \operatorname{Frac}\left(\tilde{W}_{n-1}\right) \cap W_{n-1}\left[\left[X_{n}\right]\right]=S^{-1} \tilde{W}_{n-1} .
$$

Set $\alpha=g(Y) X_{n}^{c} Z-F(Y) \in \dot{W}_{n}[Y, Z]$ then

$$
\alpha(\tilde{y}, \tilde{z})=g(\tilde{y}) X_{n}^{c} \tilde{z}-F(\tilde{y})=0 .
$$


Assertion $2 . \exists \dot{y}, \stackrel{\circ}{z}$ in $\stackrel{\circ}{W}_{n}$ so that $\alpha(\stackrel{\circ}{y}, \stackrel{\circ}{z})=0$.

Therefore $F(\stackrel{\circ}{y})=g(\stackrel{\circ}{y}) \stackrel{\circ}{z} X_{n}^{c}=\delta^{2}(\stackrel{\circ}{y}) \stackrel{\circ}{z} X_{n}^{c}$, and, since by property (e) $\left(\stackrel{\circ}{W}_{n}, X\right)$ is a Henselian pair, we can apply Tougéron's Lemma (2.2.2) in order to get $\stackrel{\circ}{x}$ in $\stackrel{\circ}{W}_{n}$ with $F(\stackrel{\circ}{x})=0$ and $\stackrel{\circ}{y} \stackrel{\circ}{x} \bmod \delta(\stackrel{\circ}{y}) X_{n}^{c}$

2.4.2. Proof of Assertion 1. Write $E_{m+1}=\stackrel{\circ}{L}\left(\bar{Y}_{1}, \ldots, \bar{Y}_{m}\right)$; then $\bar{Y}_{m+1}$ is algebraic over $E_{m+1}$ with minimal polynomial $\varphi_{m+1}(T)$. Write

$$
E_{m+2}=E_{m+1}\left(\bar{Y}_{m+1}\right) \cong \frac{E_{m+1}\left[Y_{m+1}\right]}{\left(\varphi_{m+1}\left(Y_{m+1}\right)\right)}
$$

call

$$
\gamma_{m+1}=\varphi_{m+1}\left(Y_{m+1}\right)=h_{m+1},
$$

so $\gamma_{m+1} \in \not p$.

Then $\bar{Y}_{m+2}$ is algebraic over $E_{m+2}$, with minimal polynomial $\gamma_{m+2}\left(Y_{m+2}\right)$; write

$$
E_{m+3}=E_{m+2}\left(\bar{Y}_{m+2}\right) \cong \frac{E_{m+2}\left[Y_{m+2}\right]}{\left(\gamma_{m+2}\right)} \cong \frac{E_{m+1}\left[Y_{m+1}, Y_{m+2}\right]}{\left(\gamma_{m+1}, \gamma_{m+2}\right)}
$$

since, by construction $\gamma_{m+2}\left(\bar{Y}_{m+2}\right)=0$ in $E$ we have $\gamma_{m+2} \mid \varphi_{m+2}\left(Y_{m+2}\right)$ $=h_{m+2}$ in $E_{m+2}\left[Y_{m+2}\right]$, so

$$
\exists \delta_{m+2} \in \stackrel{\circ}{W}[Y]: \gamma_{m+2} \cdot \delta_{m+2} \equiv h_{m+2} \bmod \gamma_{m+1} .
$$

Since $E / \stackrel{\circ}{L}$ is separable, $\bar{Y}_{m+2}$ is a simple root of $\varphi_{m+2}$; we therefore have $\delta_{m+2}\left(\bar{Y}_{m+2}\right) \neq 0$, i.e. $\delta_{m+2} \notin \not$.

So by induction we find the following data for $j=1, \ldots, N-m$

$$
\gamma_{m+j} \in E_{m+j}\left[Y_{m+j}\right] \quad \delta_{m+j} \in \stackrel{\circ}{W}[Y]
$$

so that

$$
\begin{gathered}
E_{m+j+1} \stackrel{\text { def }}{=} E_{m+j}\left(\bar{Y}_{m+j}\right) \cong \frac{E_{m+j}\left[Y_{m+j}\right]}{\left(\gamma_{m+j}\right)}, \\
\gamma_{m+j} \cdot \delta_{m+j} \equiv h_{m+j} \bmod \left(\gamma_{m+1}, \ldots, \gamma_{m+j-1}\right), \\
\delta_{m+j} \notin p .
\end{gathered}
$$

Therefore, by (5),

$$
E=E_{m+1}\left(\bar{Y}_{m+1}, \ldots, \bar{Y}_{N}\right) \cong \stackrel{\circ}{L}\left(\bar{Y}_{1}, \ldots, \bar{Y}_{m}\right) \frac{\left[Y_{m+1}, \ldots, Y_{N}\right]}{\left(\gamma_{m+1}, \ldots, \gamma_{N}\right)} .
$$


Now, for $j=1, \ldots, q, f_{j} \in \not p$, so $f_{j}=0$ in $E$, so

$$
f_{j}=\sum_{i=1}^{N-m} \frac{\alpha_{i j}}{\beta_{i j}} \gamma_{m+i}
$$

with $\alpha_{i j} \in \stackrel{\circ}{W}[Y]$ and $\beta_{i j} \in \stackrel{\circ}{W}\left[Y_{1}, \ldots, Y_{m}\right]$ but $\stackrel{\circ}{W}\left[Y_{1}, \ldots, Y_{m}\right] \subset$ $\stackrel{\circ}{L}\left(Y_{1}, \ldots, Y_{m}\right) \subset E$, so $\beta_{t j} \notin \not$. Call finally $\theta=\prod_{j=1}^{q} \prod_{i=1}^{N-m} \beta_{i j} \delta_{m+i} \notin \not p$ by (7).

But by (6)

$$
\theta \cdot\left(\frac{\gamma_{m+i}}{\beta_{i j}}\right) \equiv 0 \quad \bmod \left(h_{m+1}, \ldots, h_{m+l-1}\right)
$$

so

$$
\theta f_{j} \equiv 0 \bmod \left(h_{m+1}, \ldots, h_{N}\right)
$$

2.4.3. Proof of Assertion 2. Let's rewrite the statement: We know by induction that $W_{n-1}$ has A.P. over $\stackrel{\circ}{W-1}_{n}$

$$
\begin{array}{ll}
\gamma(T)=\left(\gamma_{1}, \ldots, \gamma_{K}\right), & T=\left(T_{1}, \ldots, T_{\lambda}\right), \\
\gamma_{l}(T) \in \mathcal{O}[X][T], & X=\left(X_{1}, \ldots, X_{n}\right), \\
u=\left(u_{1}, \ldots, u_{\lambda}\right), & u_{\imath} \in S^{-1}\left(W_{n-1}\left[X_{n}\right]\right),
\end{array}
$$

where $S=1+X_{n} \cdot W_{n-1}\left[X_{n}\right]$, such that $\gamma(u)=0$. We have to find

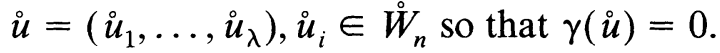

Since $u_{k} \in S^{-1}\left(W_{n-1}\left[X_{n}\right]\right)$ we can write it in the form

$$
u_{k}=\left(\sum_{i=0}^{t} \omega_{i k} X_{n}^{i}\right) /\left(1+X_{n} \cdot\left(\sum_{i=0}^{t} \theta_{\imath k} X_{n}^{i}\right)\right)
$$

$k=1, \ldots, \lambda$, with $\omega_{i k}, \theta_{i k} \in W_{n-1}$.

Set $\omega=\left(\omega_{i k}\right), \theta=\left(\theta_{i k}\right), \Omega=\left(\Omega_{i k}\right)$ and $\Theta=\left(\Theta_{i k}\right)$; write

$$
U_{k}\left(\Omega, \Theta, X_{n}\right)=\left(\sum_{i=0}^{t} \Omega_{i k} X_{n}^{i}\right) /\left(1+X_{n} \cdot\left(\sum_{i=0}^{t} \Theta_{i k} X_{n}^{i}\right)\right)
$$

so $U_{k}\left(\Omega, \Theta, X_{n}\right) \in \mathbf{Q}\left(\Theta, X_{n}\right)[\Omega]$.

Let $U=\left(U_{1}, \ldots, U_{\lambda}\right)$ and call

$$
\rho=\text { maximum of } \operatorname{deg}_{T} \gamma_{l} \text { for } l=1, \ldots, K \text {. }
$$

Finally, for $l=1, \ldots, K$, set

$$
\alpha_{l}(\Omega, \Theta, X)=\left(\prod_{j=1}^{\lambda}\left(1+X_{n} \cdot\left(\sum \Theta_{i j} X_{n}^{i}\right)\right)\right)^{\rho} \cdot \gamma_{l}(U)
$$


so that, by choice of $\rho, \alpha_{l} \in \mathcal{O}[\Omega, \Theta, X]$. Since $u_{k}=U_{k}\left(\omega, \theta, X_{n}\right)$ and $\gamma_{l}(u)=0$ we have $\alpha_{l}(\omega, \theta, X)=0$.

Write $\alpha_{l}=\sum_{k=0}^{s} \alpha_{l k} X_{n}^{k}$, with $\alpha_{l k} \in \mathcal{O}\left[\Omega, \Theta, X^{\prime}\right]$ where $X^{\prime}=$ $\left(X_{1}, \ldots, X_{n-1}\right)$, so $\alpha_{l k}\left(\omega, \theta, X^{\prime}\right)=0$ because $\omega$ and $\theta$ do not depend on $X_{n}$. By the A.P. of $W_{n-1} / \stackrel{\circ}{W}_{n-1}$ we can find $\stackrel{\circ}{\omega}, \stackrel{\theta}{\text { in } \dot{W}_{n-1}}$, such that

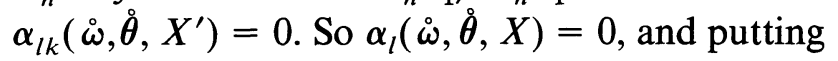

$$
\begin{aligned}
& \stackrel{\circ}{u}_{k}=U_{k}(\stackrel{\circ}{\omega}, \stackrel{\theta}{)}) \\
& =\left(\sum \stackrel{\circ}{\omega}_{i k} X_{n}^{i}\right) /\left(1+X_{n}\left(\sum \stackrel{\circ}{i k}_{i k} X_{n}^{i}\right)\right) \\
& \in\left(1+X_{n} \cdot \stackrel{\circ}{W}_{n-1}\left[X_{n}\right]\right)^{-1} \stackrel{\circ}{W}_{n-1}\left[X_{n}\right]
\end{aligned}
$$

and since $\stackrel{\circ}{W}_{n}$ forms a PP system, $\stackrel{\varkappa}{k}_{k} \in \stackrel{\circ}{W}_{n}$ and $\gamma(\stackrel{u}{u})=0$.

2.5. In this point, we will show that the C.I.C.-condition (condition (d) of 2.4.1.) automatically holds for complete rank-one valuation rings.

2.5.1. Proposition. If $R$ is a valuation ring, then $R$ is C.I.C. $\Leftrightarrow R$ is rank-one.

Proof. If $R$ is rank-one, i.e. value-group $\subset \mathbf{R}, x \in \operatorname{Frac} R, d \in R$ so that $d \cdot x^{q} \in R$ for $q=1,2, \ldots$ then is $\operatorname{ord}(d)+q \operatorname{ord}(x) \geq 0, \forall q \in \mathbf{N}$, proving that $\operatorname{ord}(x)>0$.

If $R$ is not rank-one then is the value-group $\Gamma$ non-archimedian (see [Ba]). So we can find $\alpha, \beta \in R$ with $a=\operatorname{ord}(\alpha), b=\operatorname{ord}(\beta), b>0, a \geq 0$ and $\forall q \in \mathbf{N}: a>q b$. Set $x=1 / \beta \notin R$ but $\alpha x^{q} \in R, \forall q \in \mathbf{N}$.

2.5.2. Proposition. Let $\mathcal{O}$ be a complete rank-one valuation ring and $\stackrel{\circ}{W}_{n}$ a PP-system over $\mathcal{O}$. If $\stackrel{\circ}{W}_{n}$ has properties (a), (b) and (c) of 2.4 .1 then $W_{n}$ is C.I.C. (i.e. property (d) holds).

Proof. Let $x=a / b \in \operatorname{Frac} W\left(W=W_{n}, \stackrel{\circ}{W}=\stackrel{\circ}{W}_{n}\right)$ with $a, b \in W, b$ $\neq 0$ and let $d \in W$ such that $\forall q \in \mathbf{N}: d x^{q} \in W$.

Since $W \subset \mathcal{O}[[X]]$ and this is C.I.C. by 2.5.1. and 2.1.1. we have

$$
x \in \mathcal{O}[[X]] \text {. }
$$

Set $\operatorname{ord}(b)=\varepsilon$, by property (a) (which automatically holds also for $W_{n}$ ) we can find $\pi \in \mathcal{O}$ and $\tilde{b} \in W$ so that $\operatorname{ord}(\pi)=\varepsilon$ and $b=\pi \cdot \tilde{b}$.

Since ord $a-\operatorname{ord} b=$ ord $x \geq 0$ by (1), also ord $a \geq \varepsilon$, and therefore $\exists \tilde{a} \in W: a=\pi \tilde{a}$ and thus $x=\tilde{a} / \tilde{b}$ with ord $\tilde{b}=0$. 
By (c) and early remark we may suppose $\tilde{b}$ is regular. Therefore, by (b), we find unique $q \in W$ and $r \in W_{n-1}\left[X_{n}\right]$ so that

$$
\tilde{a}=q \cdot \tilde{b}+r .
$$

But $\tilde{a}=\tilde{b} x$ and by uniqueness of the W.P.T. in $\mathcal{O}[[X]]$ (see $\S 5$ ), we find $r=0$ and $q=x$, so $x \in W$.

\section{Algebraic elements.}

3.1.1. We will call a valuation ring $A$ separative when every $f \in$ $A[[X]]^{\text {alg }}$ is separable. For example D.V.R. are separative, and by considering Newton-polygons one also can prove rank-one valuation rings are, see for instance [Ro].

3.1.2. Take $\mathcal{O}$ a complete rank-one valuation ring, with valuation ord, maximal ideal $\mathscr{M}$ and fraction field $K$. Set $X=\left(X_{1}, \ldots, X_{n}\right)$. Now take as a PP-system

$$
\stackrel{\circ}{W}_{n}=\mathcal{O}[[X]]^{\text {alg }},
$$

i.e. the ring of power series in $\mathcal{O}[[X]]$ which are algebraic over $K(X)=$ $\operatorname{Frac}(\mathcal{O}[X])$. It's easy to see it's a PP-system. Call $W_{n}=\mathcal{O}[[\hat{X}]]^{\text {alg }}=$ the closure of $\mathcal{O}[[X]]^{\text {alg }}$ in $\mathcal{O}[[X]]$ with respect to the valuation, the ring of what is called algebraic elements.

Robba proves the A.P. for $W_{n} / \stackrel{\circ}{W}_{n}$ under the condition that char $K=$ 0 . We will prove it for general characteristic.

Therefore, we have to verify the conditions of Theorem 2.4.1. Property (a) holds trivially. In $\S 5$ we will show that $\mathcal{O}[[X]]^{\text {alg }}$ has a W.P.T. (5.3.6.) and that therefore also its completion $W_{n}$ has a W.P.T. (5.5.) Property (c) holds, since it holds in $\stackrel{\circ}{W}_{n}$ as stated in 3.1.1. By Proposition 2.5.2. condition (d) automatically holds.

We have that $(\mathcal{O}[[X]],(X))$ forms a Henselian pair (because it is complete), and by using [La; X.\$7, Proposition 8] we find property (e).

So the only thing left to do is the case that char $K=p \neq 0$, and to prove that Frac $W_{n} /$ Frac $\mathscr{W}_{n}$ is separable. We will prove a stronger result, namely $\operatorname{Frac}\left(\mathcal{O}[[X]]^{\text {alg }}\right) \subset \operatorname{Frac}(\mathcal{O}[[X]])$ is separable.

3.2.1. LemMA. Let $K / k$ be algebraic, then $K^{1 / p^{\infty}}=K \cdot k^{1 / p^{\infty}}$.

REMARK. By $K^{1 / p^{\infty}}$ we mean $\left\{\alpha \in \bar{K} \mid \exists n: \alpha^{p^{n}} \in K\right\}$ where $\bar{K}$ is an algebraic closure of $K$. 
Proof.

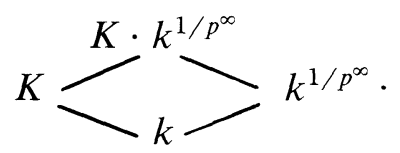

Since $K / k$ is algebraic, we have $K \cdot k^{1 / p^{\infty}} / k^{1 / p^{\infty}}$ is algebraic, but $k^{1 / p^{\infty}}$ is perfect, so $K \cdot k^{1 / p^{\infty}}$ is perfect, see for instance [La; VII §7]. Therefore, since $K$ is a subfield, $K^{1 / p^{\infty}} \subset K \cdot k^{1 / p^{\infty}}$, the other inclusion being trivial.

3.2.2. Proposition. Let $k \subset K \subset L$ be field extensions such that $K / k$ is algebraic and $L / k$ is separable. Then $L / K$ is separable.

Proof. Let $\left\{u_{\alpha}\right\}_{\alpha \in I}$ be a base of $k^{1 / p^{\infty}}$ over $k$. Since $L / k$ is separable, we know by Mac Lane's criterion (cf. [La]) that $L$ and $k^{1 / p^{\infty}}$ are linearly disjoint over $k$, i.e. $\left\{u_{\alpha}\right\}_{\alpha \in I}$ are free over L. By 3.2.1. $\left\{u_{\alpha}\right\}_{\alpha \in I}$ generate $K^{1 / p^{\infty}}$ over $K$, so $\exists J \subset I$ such that $\left\{u_{\alpha}\right\}_{\alpha \in J}$ form a base of $K^{1 / p^{\infty}} / K$, still free over $L$, so by Mac Lane's criterion again, we find $L / K$ is separable.

3.2.3. Corollary. Let $A$ be a domain; then, with $X=\left(X_{1}, \ldots, X_{n}\right)$, $\operatorname{Frac}(A[[X]]) / \operatorname{Frac}\left(A[[X]]^{\mathrm{alg}}\right)$ is separable.

Proof. Put $K=\operatorname{Frac} A$. Then $K(X) \subset K((X))=\operatorname{Frac} K[[X]]$ is separable and since Frac $A[[X]] \subset K((X))$, we find $\operatorname{Frac} A[X] \subset \operatorname{Frac} A[[X]]$ is separable. Applying 3.2.2. gives us the desired result.

We can prove easily full A.P., i.e. for arbitrary equations:

3.3. Theorem. Let $\mathcal{O}$ be a complete rank-one valuation ring. And $f=\left(f_{1}, \ldots, f_{q}\right), f_{l} \in \mathcal{O}[[X]]^{\mathrm{alg}}[Y]$

$$
X=\left(X_{1}, \ldots, X_{n}\right), \quad Y=\left(Y_{1}, \ldots, Y_{N}\right), \quad c \in \mathbf{N}
$$

and $\bar{y}=\left(\bar{y}_{1}, \ldots, \bar{y}_{N}\right), \bar{y}_{l} \in \mathcal{O}[[\hat{X}]]^{\text {alg }}$ such that $f(\bar{y})=0$. Then there exist $\dot{y}=\left(\dot{y}_{1}, \ldots, \dot{y}_{N}\right)$ in $\mathcal{O}[[X]]^{\text {alg }}$ s.t. $f(X, \dot{y})=0$ and $\bar{y} \equiv \dot{y} \bmod (X)^{c}$.

Proof. Write $f_{i}=\sum_{j \in J} \alpha_{\imath j} Y^{J}$ with $\alpha_{\imath} \in \mathcal{O}[[X]]^{\text {alg }}$ and $J$ a finite set of multi-indices $j=\left(j_{1}, \ldots, j_{N}\right)$. So there exist $\gamma_{i j}\left(X, T_{i j}\right) \in \mathcal{O}[X]\left[T_{i j}\right]$ for $i=1, \ldots, q, j \in J$ such that $\gamma_{i j}\left(X, \alpha_{i j}\right)=0$. Take $c$ big enough so that the distinct roots in $\mathcal{O}[[X]]$ of each $\gamma_{i j}$ (considered as equation in $T_{i j}$ ) are 
not congruent $\bmod (X)^{c}$. Call

$$
F_{i}=\sum_{j \in J} T_{i j} Y^{j}, \quad F=\left(F_{1}, \ldots, F_{q}\right)
$$

and $\gamma=\left(\gamma_{i j} \mid i=1, \ldots, q, j \in J\right), T=\left(T_{i j} \mid i=1, \ldots, q, j \in J\right)$. Consider the system of polynomial equations $(F, \gamma)$ in $(Y, T)$ over $\mathcal{O}[X]$ and solution $\left(\bar{y}, \alpha_{i j}\right)$ in $\mathcal{O}[[\hat{X}]]^{\text {alg }}$. By the A.P. already proved, there exists $\dot{y}, \stackrel{\circ}{\alpha}_{i j}$ in $\mathcal{O}[[X]]^{\text {alg }}$ so that they are roots of $(F, \gamma)$ and $\alpha_{i j} \equiv$ $\stackrel{\circ}{\alpha}_{i j} \bmod (X)^{c}, \bar{y} \equiv \dot{y} \bmod (X)^{c}$. Since $\gamma_{i j}\left(\stackrel{\circ}{\alpha}_{i j}\right)=0$ we get by choice of $c$ that actually $\stackrel{\circ}{\alpha}_{i j}=\alpha_{i j}$. Therefore

$$
0=F\left(\stackrel{\circ}{\alpha}_{i j}, \stackrel{\circ}{)}\right)=F\left(\alpha_{i j}, \stackrel{\circ}{y}\right)=f(\stackrel{\circ}{y} \text {. }
$$

\section{Henselian elements.}

4.1.1. Let $A$ be an arbitrary domain, $X=\left(X_{1}, \ldots, X_{n}\right)$ and $Y$ one variable. We will define a sort of diagonalisation operator $\mathscr{D}$ by

$$
\begin{aligned}
\mathscr{D}: A[[X, Y]] & \rightarrow A[[X]], \\
\sum a_{i j} X^{i} Y^{j} & \mapsto \sum_{i_{1}+i_{2}+\cdots+i_{n}=j} a_{i j} X^{i},
\end{aligned}
$$

where for $i=\left(i_{1}, \ldots, i_{n}\right)$ a multi-index we mean by $X^{i}=X_{1}^{i_{1}} \cdot X_{2}^{i_{2}} \cdots X_{n}^{i_{n}}$. Clearly $\mathscr{D}$ is $A$-linear.

4.1.2. Theorem [Lipshitz, Denef]. Let $A$ be an excellent local integral domain, $f \in A[[X]]$, then

$$
\begin{aligned}
f & \in A[[X]]^{\text {alg }} \\
& \Leftrightarrow \exists R \in A[[X, Y]] \cap \operatorname{Frac}(A[X, Y]) \text { s.t. } f=\mathscr{D}(R) .
\end{aligned}
$$

In words, $f$ is algebraic if and only if it is the diagonal of a rational power series.

Proof. See [L.D.; Thm. 6.2].

4.2.1. In this paragraph we will restrict ourselves to a special type of rank-one valuation rings. Start with a complete D.V.R. $\tilde{\mathcal{O}}$, with local parameter $\tau$ and residue field $\tilde{k}$, fraction field $\tilde{K}=$ Frac $\tilde{\mathcal{O}}$. Let $\bar{K}$ be an algebraic extension of $\tilde{K}$; then the discrete valuation extends in $\bar{K}$ uniquely to a rank-one valuation, ord. Let $K$ be the completion of $\bar{K}$ with respect to this valuation and $\mathcal{O}$ the ring of integers in $K$. So $\mathcal{O}$ is a complete rank-one valuation ring, with valuation ord. Let $k$ be the residue 
field. By the theory of Witt-rings (see [Se]) we know that there is a complete D.V.R. $W(k)$ in $\mathcal{O}$, which contains $\tilde{\mathcal{O}}$, so we could have started with this D.V.R. to obtain $\mathcal{O}$, and therefore we may suppose as well that the residue field of $\tilde{\mathcal{O}}$ is equal to $k$ from the start but we only will need this in char $>0$. We make one final restriction on $\mathcal{O}$ : we demand that $K$ be perfect. So, for char $K=p$, we need that $\mathcal{O}=\mathcal{O}^{1 / p}$. Remark that in this case $k$ also is perfect (this is not necessary though when char $K=0$ ). Note also that the value-group of $K$ is a subgroup of $\mathbf{Q},+$, so $\mathcal{O}$ might be non-Noetherian. Example of a $\mathcal{O}: \mathcal{O}=\mathcal{O}_{C_{p}}$, the ring of integers in the $p$-adic closure of the algebraic closure of $\mathbf{Q}_{p}$.

4.2.2. THEOREM. Let $\mathcal{O}$ be as above (the restriction on the perfectness is not needed) and $f \in \mathcal{O}[[X]]^{\text {alg }}$. Then $\exists f_{n} \in \mathcal{O}[[X]]^{\text {alg }}, f_{n}$ integral coefficients in a finite extension of $\tilde{K}$ such that

$$
f_{n} \rightarrow f \text {. }
$$

Since by 4.1.2 $\exists t_{n} \in \mathcal{O}[[X, Y]] \cap \operatorname{Frac} \mathcal{O}[X, Y]$ such that $f_{n}=\mathscr{D}\left(t_{n}\right)$, the above theorem is a generalisation of a result of Christol in one variable: " $f$ is a uniform limit of "fonctions algébrique régulières" see [Chr].

Proof. Since $f$ is algebraic over $\operatorname{Frac} \mathcal{O}[X]=K(X)$ we know by Theorem 4.1.2

$$
\exists \alpha, \beta \in K[X, Y], \quad \theta=\alpha / \beta \in K[[X, Y]]
$$

such that $f=\mathscr{D}(\theta)$.

Since $K[[X]]$ is faithfully flat over $K[X]_{(X)}$, we even may suppose that $\beta(0)=1$.

Let's write $Z=\left(X_{1}, \ldots, X_{n}, Y\right)$ and write

$$
\begin{aligned}
& \alpha=\sum_{i \in I} a_{i} Z^{i}, \\
& \beta=\sum_{i \in I} b_{i} Z^{i},
\end{aligned}
$$

Write $A=\left(A_{i} \mid i \in I\right)$ and $B=\left(\left.B_{i}\right|_{i \neq 0} i \in I\right)$ and

$$
\begin{aligned}
& \tilde{\alpha}(A, Z)=\sum_{i \in I} A_{i} Z^{i}, \\
& \tilde{\beta}(B, Z)=\sum_{\substack{i \in I \\
i \neq 0}} B_{i} Z^{i}+1, \quad \tilde{\boldsymbol{\alpha}}, \tilde{\beta} \in \mathbf{Z}[A, B, Z],
\end{aligned}
$$

and $\tilde{\theta}(A, B, Z)=\tilde{\alpha} / \tilde{\beta} \in \mathbf{Z}[[A, B, Z]]$ since $\tilde{\beta}(0)=1$. 
Even more, we can write

$$
\tilde{\boldsymbol{\theta}}=\sum_{j} \boldsymbol{\theta}_{j}(A, B) Z^{j} \in \mathbf{Z}[A, B][[Z]]
$$

where $\theta_{J} \in \mathbf{Z}[A, B]$ with total degree in $A$ and $B$ less than or equal to $1+\|j\|$.

Now, write $a=\left(a_{i} \mid i \in I\right)$ and $b=\left(b_{i} \mid i \in I, i \neq 0\right)$ so

$$
\tilde{\alpha}(a, Z)=\alpha, \quad \tilde{\beta}(b, Z)=\beta, \quad \tilde{\theta}(a, b, Z)=\theta .
$$

By the construction of $\mathcal{O}$, we can find for each $n \in \mathbf{N}_{0}$ a field $K^{(n)}$, finite over $\tilde{K}$ and $\forall i \in I: a_{i}^{(n)}, b_{i}^{(n)} \in K^{(n)}$ with $b_{0}^{(n)}=1$, such that

$$
\operatorname{ord}\left(a_{i}-a_{i}^{(n)}\right), \quad \operatorname{ord}\left(b_{i}-b_{i}^{(n)}\right) \geq n .
$$

Call

$$
\begin{aligned}
& \alpha_{n}(Z)=\tilde{\alpha}\left(a_{i}^{(n)}, Z\right), \quad \tilde{\alpha}, \tilde{\beta} \in K^{(n)}[Z] . \\
& \beta_{n}(Z)=\tilde{\beta}\left(b_{i}^{(n)}, Z\right),
\end{aligned}
$$

So $\alpha_{n} \rightarrow \alpha, \beta_{n} \rightarrow \beta$ for $n \rightarrow \infty$.

Finally, call

$$
t_{n}(Z)=\theta\left(a_{i}^{(n)}, b_{i}^{(n)}, Z\right) \in K^{(n)}[[Z]]=\alpha_{n} / \beta_{n}
$$

so $t_{n}$ is a rational power series and one may check that

$$
t_{n} \rightarrow \theta \text {. }
$$

Let us call $f_{n}=\mathscr{D}\left(t_{n}\right)$, so $f_{n} \in K^{(n)}[[X]]$, and by 4.1 .2 we know that it is algebraic.

Since $f=\mathscr{D}(\theta)$ and (3) we get

$$
f_{n} \rightarrow f
$$

So, for $n$ big enough, $f_{n} \in \mathcal{O}[[X]]$ since $f$ is.

Call $\mathcal{O}^{(n)}$ the ring of integers in $K^{(n)}$. This is a D.V.R. and hence $K^{(n)} \cap \mathcal{O}=\mathcal{O}^{(n)}$, and so

$$
f_{n} \in \mathcal{O}^{(n)}[[X]]^{\text {alg }} \text {. }
$$

4.2.3. CoROllaRY. When $\mathcal{O}_{\bar{K}}$ denotes the ring of integers in $\bar{K}$ we get:

$$
\mathcal{O}_{\bar{K}}[[\hat{X}]]^{\text {alg }}=\mathcal{O}[[\hat{X}]]^{\text {alg }} \text {. }
$$

REMARK. We also have proved explicitly that $\mathcal{O}$ is separative.

\subsubsection{Let's write}

$\mathscr{A}=\{A \operatorname{ring} \mid \tilde{\mathcal{O}} \subset A \subset \mathcal{O}$ and $A$ finitely generated $\tilde{\mathscr{O}}$-algebra $\}$. 
We define now

$$
\stackrel{\circ}{R}_{\left[\left[X_{1}, \ldots, X_{n}\right]\right]}=\bigcup_{A \in \mathscr{A}} A\left[\left[X_{1}, \ldots, X_{n}\right]\right] ;
$$

we will mostly abbreviate this as $\stackrel{\circ}{R}_{n}$ or $\stackrel{\circ}{R}$. Denote by $R_{[[X]]}$ the completion of $\stackrel{\circ}{R}_{[[X]]}$ in $\mathcal{O}[[X]]$ with respect to ord.

Again, $\stackrel{R}{R}_{n}$ forms a PP-system. We now verify the conditions of Theorem 2.4.1 in order to get A.P. Since each $A \in \mathscr{A}$ is noetherian, each element of $\stackrel{\circ}{R}$ is separable, and therefore, also of $R$, which proves (c). Property (b) will be postponed to $\S 5$ (5.4.2.); (d) already is dealt with by 2.5.2. Also, each $(A[[X]],(X))$ for $A \in \mathscr{A}$ is a Henselian pair, and therefore also the direct limit $(\stackrel{\circ}{R},(X))$, proving (e).

4.3.2. LEMMA. $\forall \pi \in \mathcal{O}, \quad \operatorname{ord}(\pi)>0: \pi \mathcal{O}[[X]] \cap \stackrel{\circ}{R}=\pi \stackrel{\circ}{R}$.

Proof. Choose $\gamma \in \pi \mathcal{O}[[X]] \cap \stackrel{\circ}{R}$ and $A \in \mathscr{A}$ s.t. $\gamma \in A[[X]]$ and let

(1) $a_{1}, \ldots, a_{s}$ be generators of $A$ over $\tilde{\mathcal{O}}$, so $A=\tilde{\mathcal{O}}\left[a_{1}, \ldots, a_{s}\right]$.

Therefore

$$
\begin{aligned}
& \exists c_{1}, \ldots, c_{s} \in \mathcal{O}_{\bar{K}}(\text { integers of } \bar{K}) \\
& \exists d_{1}, \ldots, d_{s} \in \mathcal{O}
\end{aligned}
$$

s.t.

$$
a_{\imath}=c_{\imath}+\pi d_{\imath} .
$$

Let $F=\tilde{K}\left(c_{1}, \ldots, c_{s}\right) \subset \bar{K}$, so $F$ is a finite extension of $\tilde{K}$. Let $\mathcal{O}_{F}$ be the ring of integers then we have, see [Ma; (31.C)]

$$
\begin{aligned}
& \mathcal{O}_{F} \text { is a D.V.R. } \\
& \mathcal{O}_{F} \text { finite, and therefore free } \tilde{\mathcal{O}} \text {-module. }
\end{aligned}
$$

Let $e_{1}, \ldots, e_{w}$ be a basis of $\mathcal{O}_{F}$ over $\tilde{\mathcal{O}}$ and set

$$
\tilde{A}=\tilde{\mathcal{O}}\left[e_{1}, \ldots, e_{w}, d_{1}, \ldots, d_{s}, \pi\right]
$$

so $\mathcal{O}_{F} \subset \tilde{A}$ and by (2) also $A \subset \tilde{A}$.

We claim that $\gamma / \pi \in \tilde{A}[[X]]$, so is in $\stackrel{R}{R}$. Indeed, let $c$ be a coefficient of $\gamma$, so $c \in A$, and $c \equiv 0 \bmod \pi$ and by (1)

$$
c=\mu\left(a_{1}, \ldots, a_{s}\right)
$$

with $\mu\left(Y_{1}, \ldots, Y_{s}\right) \in \tilde{\mathcal{O}}[Y]$.

By (2) we can write

$$
c=\mu\left(c_{1}, \ldots, c_{s}\right)+\pi \lambda\left(c_{1}, \ldots, c_{s}, d_{1}, \ldots, d_{s}, \pi\right)
$$


where $\lambda\left(Y_{1}, \ldots, Y_{s}, Z_{1}, \ldots, Z_{s}, T\right) \in \tilde{\mathcal{O}}[Y, Z, T]$ so $\lambda=$ $\lambda\left(c_{1}, \ldots, c_{s}, d_{1}, \ldots, d_{s}, \pi\right) \in \tilde{A}$ and

$$
\frac{c}{\pi}=\frac{\mu\left(c_{1}, \ldots, c_{s}\right)}{\pi}+\lambda \in \mathcal{O},
$$

so $\mu\left(c_{1}, \ldots, c_{s}\right) / \pi \in \mathcal{O}$ and $\mu\left(c_{1}, \ldots, c_{s}\right) \in \mathcal{O}_{F}$, which by (3) is a D.V.R., therefore $\mu\left(c_{1}, \ldots, c_{s}\right) / \pi \in \mathcal{O}_{F} \subset \tilde{A}$ and therefore by (4) finally, $c / \pi \in \tilde{A}$.

4.3.3. Lemma. Call $L=\operatorname{Frac} \mathcal{O}[[X]]$ and $\stackrel{\circ}{K}=\operatorname{Frac} \stackrel{\circ}{R}$ then $L / \stackrel{\circ}{K}$ is separable.

Proof. Suppose char $K=p \neq 0$.

We have to prove that $L$ and $\stackrel{\circ}{K}^{1 / p}$ are linearly disjoint. Let $\left\{e_{1}, \ldots, e_{n}\right\}$ be free over $\stackrel{\circ}{K}, e_{i} \in L$. We have to show that they remain free over $\stackrel{\circ}{K}^{1 / p}$. So, suppose $\exists \alpha_{\imath} \in \stackrel{\circ}{K}^{1 / p}$

$$
\sum_{i=1}^{m} \alpha_{i} e_{i}=0
$$

We may assume that $e_{i} \in \mathcal{O}[[X]]$ and $\alpha_{l} \in \stackrel{\circ}{R}^{1 / p}$. Let $\rho_{i}=\alpha_{i}^{p} \in \stackrel{\circ}{R}$ and $A \in \mathscr{A}$ s.t. $\rho_{i} \in A[[X]]$. Write out

$$
\rho_{i}=\sum \rho_{i j} X^{j}, \quad \rho_{i j} \in A .
$$

Let $\nu_{i j}=\rho_{i j}^{1 / p} \in A^{1 / p}$ and call $\delta_{i}=\sum \nu_{i j} X^{j}$. So we find that

$$
\left(\delta_{i}(X)\right)^{p}=\alpha_{i}^{p}\left(X^{p}\right) .
$$

Therefore, $\delta_{l}(X)=\alpha_{i}\left(X^{p}\right) \in A^{1 / p}[[X]]$.

Assertion. $A^{1 / p} \in \mathscr{A}$.

Therefore $\delta_{i}=\alpha_{l}\left(X^{p}\right) \in \stackrel{\circ}{R}$ and from (1) we get

$$
\sum \delta_{i}(X) \cdot e_{i}\left(X^{p}\right)=0 .
$$

Write out $\delta_{i}$ in $p$-basis, i.e.

$$
\delta_{i}=\sum \delta_{i j}\left(X^{p}\right) X^{j}
$$

where $j=\left(j_{1}, \ldots, j_{n}\right)$ varies within the range $0 \leq j_{k} \leq p-1$ and the $\delta_{i j} \in \stackrel{\circ}{R}$ since the $\delta_{i}$ are.

Substituting this in (2) gives

$$
\sum_{i, j} \delta_{i j}\left(X^{p}\right) e_{i}\left(X^{p}\right) X^{j}=0
$$


SO

$$
\forall j: \sum_{i} \delta_{i j}\left(X^{p}\right) e_{i}\left(X^{p}\right)=0
$$

so

$$
\forall j: \sum_{i} \delta_{i j} e_{i}=0
$$

and $\left\{e_{i}\right\}$ free over $\stackrel{\circ}{K}$, so $\delta_{i j}=0$, hence $\delta_{i}=0$, hence $\alpha_{i}=0$.

Proof of the assertion. Since $k=\tilde{k}$ is perfect, and $\tilde{\mathcal{O}}$ is complete, we get by Cohen's structure theorem [Co] that $\tilde{\mathcal{O}}=\tilde{k}[[T]], T$ one variable (corresponding to the local parameter $\tau$ ); therefore, since $(\tilde{k}[[T]])^{1 / p}=$ $\tilde{k}[[T]]\left[T^{1 / p}\right]$ since $\tilde{k}$ perfect we find that $\tilde{\mathcal{O}}^{1 / p}=\tilde{\mathcal{O}}\left[\tau^{1 / p}\right] \subset \mathcal{O}$, since $\mathcal{O}$ perfect. Let $A \in \mathscr{A}$ be generated over $\tilde{\mathcal{O}}$ by $u_{1}, \ldots, u_{s}$. Then $A^{1 / p}=$ $\tilde{\mathcal{O}}\left[\tau^{1 / p}, u_{1}^{1 / p}, \ldots, u_{s}^{1 / p}\right] \subset \mathcal{O}$ so $A^{1 / p} \in \mathscr{A}$.

Since Lemma 4.3.2 is nothing but property (a) of Theorem 2.4.1 and Lemma 4.3.3 implies property (f), we may conclude:

4.3.4. THEOREM. Let $\mathcal{O}, \stackrel{\circ}{R}$ and $R$ be as described above, then $R$ has A.P. over $\stackrel{\circ}{R}$ with respect to polynomial equations.

4.4.1. THEOREM. Let $\mathcal{O}$ be as before, denote with $\mathcal{O}[[X]]^{h}$ the henselisation of $\mathcal{O}[X]$ in $\mathcal{O}[[X]]$ with respect to $(X)$. Then we have

$$
\mathcal{O}[[X]]^{h}=\mathcal{O}[[X]]^{\text {alg }} \text {. }
$$

Proof. $\mathcal{O}[[X]]^{h} \subset \mathcal{O}[[X]]^{\text {alg }}$ is clear by an already stated proposition of Lang ([La; $X, \S 7$, Prop. 8]). Let $y \in \mathcal{O}[[X]]^{\text {alg; }}$ then by Theorem 4.2.2. $y$ belongs to $R=R_{[[X]]}$.

Let $P(X, T) \in \mathcal{O}[X][T]$ be a polynomial in one variable such that $P(X, y)=0$.

Choose $c$ big enough so that the distinct roots of $P(X, T)$ are not congruent $\bmod (X)^{c}$.

Applying the A.P. for $R / \stackrel{\circ}{R}$ we get a $\dot{y} \in \stackrel{\circ}{R}$ s.t.

$$
P(X, \stackrel{\circ}{y})=0 \wedge y \equiv \dot{y} \bmod (X)^{c} .
$$

By the choice of $c$ we need to have $y=\stackrel{\circ}{y}$, so actually $y \in \stackrel{\circ}{R}$. Suppose therefore that $y \in A[[X]]$ with $A \in \mathscr{A}$, i.e. finitely generated $\tilde{\mathcal{O}}$-algebra. 
Take $A$ big enough so that $P(X, T) \in A[X, T]$; then $y \in A[[X]]^{\text {alg }}$. Since $\tilde{\mathcal{O}}$ is excellent, so is $A$ and by a well-known fact for excellent domains $[\mathbf{R a}]: A[[X]]^{\text {alg }}=A[[X]]^{h}$ and since by the universal property $A[[X]]^{h} \subset$ $\mathcal{O}[[X]]^{h}$, we find

$$
y \in \mathcal{O}[[X]]^{h}
$$

\subsubsection{COROllary. $\mathcal{O}[[X]]^{\text {alg }} \subset \stackrel{\circ}{R}$.}

So, each algebraic element has coefficients which lie in a finitely generated $\tilde{\mathcal{O}}$-algebra.

\section{Weierstrass preparation theorems.}

5.1.1. Let $A$ be a ring, $a$ an ideal in $A$. Assume that $A$ is Hausdorff in its $a$-topology, i.e. $\bigcap_{n=0}^{\infty} a^{n}=0$. We shall in the sequel write $X=$ $\left(X_{1}, \ldots, X_{n}\right)$ and $X^{\prime}=\left(X_{1}, \ldots, X_{n-1}\right)$. When $f \in A[[X]]$ we call $f$ arregular in $X_{n}$ of degree $k$ if there is a term $c X_{n}^{k}$ of $f$ with $c$ a unit mod $a$ and $k$ minimal with this property. Or equivalently if $f \equiv v\left(X_{n}\right) \cdot X_{n}^{k} \bmod \left(a, X^{\prime}\right)$ with $v\left(X_{n}\right) \in A\left[\left[X_{n}\right]\right]$ and a unit $\bmod a$, i.e. a unit in $(A / a)\left[\left[X_{n}\right]\right]$.

We call $P \in A\left[\left[X^{\prime}\right]\right]\left[X_{n}\right]$ a distinguished polynomial (DP) in $X_{n}$ of degree $k$ if $P$ is of the form

$$
P=X_{n}^{k}+\sum_{i=0}^{k-1} p_{i} X_{n}^{i}
$$

with $p_{i} \in\left(a, X^{\prime}\right) A\left[\left[X^{\prime}\right]\right]$.

Recall that we shall mean by saying that a PP-system $\stackrel{\circ}{W}_{n}$ has a W.P.T. with respect to $a$ if the following holds:

$\forall f \in \stackrel{\circ}{W}_{n}$ which is a-regular in $X_{n}$ of degree $k$ and $\forall g \in \stackrel{\circ}{W}_{n}$ there are unique $q \in \stackrel{\circ}{W}_{n}, r \in \stackrel{\circ}{W}_{n-1}\left[X_{n}\right]$ s.t. $g=q \cdot f+r$ and $\operatorname{deg}_{X_{n}} r<k$.

5.1.2. LemMA. Suppose $f \in A[[X]]$ is a-regular in $X_{n}$ of degree $k$ and that there are $\beta \in A[[X]], \beta_{i} \in A\left[\left[X^{\prime}\right]\right]$ such that $\beta f \equiv \sum_{i=0}^{k-1} \beta_{i} X_{n}^{i} \bmod \left(X^{\prime}\right)$. Then $\beta \equiv \beta_{i} \equiv 0 \bmod \left(X^{\prime}\right)$.

Proof. We can write $f \equiv \varphi+X_{n}^{k} v \bmod \left(X^{\prime}\right)$ where $v \in A\left[\left[X_{n}\right]\right]$ is a unit $\bmod a$ and $\varphi \in A\left[X_{n}\right]$ with $\varphi \equiv 0 \bmod a$ and $\operatorname{deg} \varphi<k$. So, putting 
$X^{\prime}=0$,

$$
\beta\left(0, X_{n}\right) \cdot\left(\varphi+X_{n}^{k} v\right)=\sum_{i=0}^{k-1} \beta_{i}(0) X_{n}^{i}, \quad \text { thus }
$$

$$
\begin{aligned}
& \beta\left(0, X_{n}\right) X_{n}^{k} v \equiv \sum_{i=0}^{k-1} \beta_{i}(0) X_{n}^{i} \bmod a, \text { so } \\
& \beta_{i}(0) \equiv 0 \equiv \beta\left(0, X_{n}\right) \cdot v \bmod a,
\end{aligned}
$$

but $v$ unit $\bmod a$, so $\beta\left(0, X_{n}\right) \equiv 0 \bmod a$.

Substituting in (1) gives

$$
\beta\left(0, X_{n}\right) X_{n}^{k} v \equiv \sum \beta_{i}(0) X_{n}^{l} \bmod a^{2}
$$

which again implies $\beta\left(0, X_{n}\right) \equiv \beta_{i}(0) \bmod a^{2}$, and by induction $\beta\left(0, X_{n}\right)$, $\beta_{i}(0) \in \bigcap_{k=0}^{\infty} a^{k}=0$.

5.2.1. Theorem. Let $A$ be a ring, a an ideal in $A$ and suppose $A$ is complete and Hausdorff in its a-topology. Then $A[[X]]$ has a W.P.T.

Proof. Given $f \in A[[X]]$, a-regular in $X_{n}$ of degree $k, g \in A[[X]]$. Write $f \equiv \varphi+X_{n}^{k} v \bmod X^{\prime}$ where $\varphi$ polynomial in $X_{n}$ of degree less than $k$, coefficients in $a$ and $v$ a unit in $A\left[\left[X_{n}\right]\right]$. Set $E=A[[X]] /(f)$. We will show that $E$ is finitely generated as an $A\left[\left[X^{\prime}\right]\right]$-module by $1, X_{n}, \ldots, X_{n}^{k-1}$.

Set $\mathscr{N}=\left(a, X^{\prime}\right)$, so $A\left[\left[X^{\prime}\right]\right]$ is complete and Hausdorff with respect to $\mathscr{N}$.

Assertion. $E$ is Hausdorff in the $\mathcal{N}$-topology.

Since

$$
\frac{E}{\mathscr{N} E}=\frac{A[[X]]}{\left(f, a, X^{\prime}\right)}=\frac{A\left[\left[X_{n}\right]\right]}{\left(a, \varphi+X_{n}^{k} v\right)}=\frac{(A / a)\left[\left[X_{n}\right]\right]}{\left(X_{n}^{k} v\right)}
$$

with $v$ unit $\bmod a$ we find that $E / \mathscr{N} E$ is finitely generated as an $A / a$

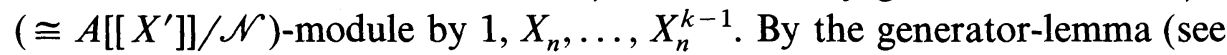
[Z-S; VIII Thm. 7, Cor. 2]) $E$ is finitely generated over $A\left[\left[X^{\prime}\right]\right]$ by $1, X_{n}, \ldots, X_{n}^{k-1}$ as module. So, we know that $\forall g \in A[[X]]$ there exist $q \in A[[X]], r \in A\left[\left[X^{\prime}\right]\right]\left[X_{n}\right], \operatorname{deg}_{X_{n}} r<k$ s.t. $g=f \cdot q+r$.

We have to show finally that $q$ and $r$ are unique. Suppose we have also $q^{\prime}$ and $r^{\prime}$ s.t. $g=f \cdot q^{\prime}+r^{\prime}$. Call $\alpha=q-q^{\prime}$ and $\beta=r^{\prime}-r$, so

$$
f \alpha=\beta=\sum_{i=0}^{k-1} \beta_{i} X_{n}^{i}, \quad \beta_{i} \in A\left[\left[X^{\prime}\right]\right] .
$$

By Lemma 5.1.2. we get $\alpha, \beta \equiv 0 \bmod X^{\prime}$. 
We will prove by induction that $\alpha, \beta \equiv 0 \bmod \left(X^{\prime}\right)^{t}$, so are zero by the Hausdorffness.

Let $\left\{\mu_{j}\right\}_{j=1}^{\lambda}$ be all monomials in $X^{\prime}$ of degree $t$ and suppose $\alpha$, $\beta \equiv 0 \bmod \left(X^{\prime}\right)^{t}$, so $\exists K_{j} \in A[[X]]$ and $\exists K_{i j} \in A\left[\left[X^{\prime}\right]\right]$ s.t. $\alpha=\sum_{j=1}^{\lambda} K_{j} \mu_{j}$ and $\beta_{i}=\sum_{j=1}^{\lambda} K_{i j} \mu_{j}$. By (2),

$$
\begin{gathered}
\left(\sum K_{j} \mu_{j}\right) f=\sum_{i}\left(\sum_{j} K_{i j} \mu_{j}\right) X_{n}^{i}=\sum_{j}\left(\sum_{i} K_{i j} X_{n}^{i}\right) \mu_{j}, \quad \text { so } \\
\forall j: K_{j} f \equiv \sum_{i=0}^{k-1} K_{i j} X_{n}^{i} \bmod X^{\prime}
\end{gathered}
$$

and by 5.1.2. again $K_{j}, K_{i j} \in\left(X^{\prime}\right)$, so $\alpha, \beta \equiv 0 \bmod \left(X^{\prime}\right)^{t+1}$.

Proof of the assertion. Let $u \in \bigcap_{i=0}^{\infty} \mathcal{N}^{i} E, u \in A[[X]]$. So

$$
\exists \alpha_{i} \in A[[X]], \quad \theta_{i} \in \mathscr{N}^{i}: u=\alpha_{i} f+\theta_{i} .
$$

For $j \geq i$ we have $\left(\alpha_{i}-\alpha_{j}\right) f \in \mathscr{N}^{i}$, but one can easily show that $f$ is a non-zerodivisor mod $\mathscr{N}^{i}$, since $f$ is regular, so $\alpha_{i}-\alpha_{j} \in \mathscr{N}^{i}$. Therefore the $\left(\alpha_{i}\right)_{i}$ form a Cauchy-sequence in the $\mathscr{N}$-topology in $A[[X]]$. By completeness, $\exists \alpha \in A[[X]]: \alpha_{i} \rightarrow \alpha$, and since $\theta_{i} \rightarrow 0$, we find $u=\alpha f$ (remark that $A[[X]]$ is Hausdorff in the $\mathscr{N}$-topology, so ' $\rightarrow$ ' makes sense) and so $u=0$ in $E$.

5.2.2. COROllaRY. With the assumptions and notations of 5.2.1. and moreover, $a \subset \operatorname{rad} A$, then $\exists$ unique $P_{f}$ and $u$ in $A[[X]]$ s.t.

$$
f=u \cdot P_{f}
$$

with $P_{f}$ a DP of degree $k$ in $X_{n}$ and $u$ a unit in $A[[X]]$.

Proof. Apply W.P.T. to $X_{n}^{k}$.

5.3.1. Let $A$ be a domain, $a$ an ideal in $A, a \subset \operatorname{rad} A$ and suppose $A[[X]]$ has a W.P.T. with respect to $a$. We want to study W.P.T. for $A[[X]]^{\text {alg }}$, extending methods of Coste-Roy [C-R] and Lafon [Laf]. Take $P$ a DP of degree $k$ in $X_{n}$ and $y \in F=$ algebraic closure of Frac $A\left[\left[X^{\prime}\right]\right]$, s.t. $P\left(X^{\prime}, y\right)=0$. By the W.P.T. $\forall g \in A[[X]]: \exists$ unique $q \in A[[X]]$, $r \in A\left[\left[X^{\prime}\right]\right]\left[X_{n}\right]\left(\operatorname{deg}_{X_{n}} r<k\right)$ s.t.

$$
g=q P+r
$$

Define a $A$-algebra morphism from $A[[X]]$ to $F$ by substitution defined as

$$
g\left(X^{\prime}, y\right) \stackrel{\text { def }}{=} r\left(X^{\prime}, y\right)
$$


If moreover, $g \in A[[X]]^{\mathrm{alg}}$ and $g\left(X^{\prime}, y\right)=0$ then one checks that $y$ is algebraic over Frac $A\left[X^{\prime}\right]$. Write $P=X_{n}^{k}+\sum_{i=0}^{k-1} p_{i} X_{n}^{i}$ and suppose $\exists h \in$ $A[[X]]$ s.t. $h P \in A[[X]]^{\text {alg }}$. Then $\forall i: p_{i} \in A\left[\left[X^{\prime}\right]\right]^{\text {alg }}$, so $h, P \in A[[X]]^{\text {alg }}$. This is because each root of $P$ is a root of $h P$, so is algebraic over Frac $A\left[X^{\prime}\right]$, but the $p_{i}$ are symmetric functions in the roots. Therefore, using this on 5.2.2 we get when $f \in A[[X]]^{\text {alg }}$ of that corollary, then also $u, P_{f} \in A[[X]]^{\mathrm{alg}}$.

5.3.2. Let $A$ be a domain of $\operatorname{char} A=p \neq 0$ and $T$ be one variable. When we put $q=p^{r}\left(r \in \mathbf{N}_{0}\right)$ we can expand each $f \in A[[T]]$ in $q$-base uniquely as follows

$$
f=\sum_{i=0}^{q-1} f_{i}\left(T^{q}\right) T^{i}
$$

Define $\Delta(f)=\sum a_{i}^{p} T^{i}$ where $f=\sum a_{i} T^{i}$, so

$$
f(T)^{p}=\Delta(f)\left(T^{p}\right) .
$$

One easily checks that $\Delta$ is an injective ring morphism and that $f \in A[[T]]^{\text {alg }}$ iff $\Delta(f) \in A[[T]]^{\text {alg }}$.

5.3.3. Proposition. Let $f \in A[[T]]^{\mathrm{alg}}$, and $f=\sum_{i=0}^{p-1} f_{i}\left(T^{p}\right) T^{i}$. Then $f_{i}(T) \in A[[T]]^{\mathrm{alg}}$.

Proof. Call $E=\operatorname{Frac} A[T]$. Then $E(f) / E$ is finite so there exist $\alpha_{j}(T) \in A[T]:$

$$
\sum_{j=r}^{s} \alpha_{j}(T) f^{p^{\prime}}=0, \quad \alpha_{r} \neq 0
$$

Set $\alpha=-\alpha_{r}$; then

$$
\alpha f^{p^{r}}=\sum_{j=r+1}^{s} \alpha_{j} f^{p^{j}}
$$

Call $q=p^{r+1}$ and multiply (1) by $\alpha^{q-1}$

$$
\alpha^{q} \cdot f^{p^{r}}=\sum_{j=r+1}^{s} \alpha^{q-1} \alpha_{j} f^{p^{j}}
$$

Let $\tilde{f}=\Delta^{r+1} f$, so $f^{q}=\tilde{f}\left(T^{q}\right)$ and $\tilde{\alpha}=\Delta^{r+1} \alpha$, so $\alpha^{q}=\tilde{\alpha}\left(T^{q}\right)$ and also $f^{p^{r}}=\sum_{i=0}^{p-1} f_{i}\left(T^{p}\right)^{p^{r}} T^{i p^{r}}$ set $\tilde{f}_{i}=\Delta^{r} f_{i}$, so $f_{i}\left(T^{p}\right)^{p^{r}}=\tilde{f}_{i}\left(T^{q}\right)$. Develop $\alpha^{q-1} \alpha_{J}=\sum_{i=0}^{q-1} \alpha_{j i}\left(T^{q}\right) T^{i}$ so substituting this all in (2) we get

$$
\sum_{i=0}^{p-1} \tilde{\alpha}\left(T^{q}\right) \tilde{f}_{i}\left(T^{q}\right) T^{i p^{r}}=\sum_{i=0}^{q-1} \sum_{j=r+1}^{s} \alpha_{j i}\left(T^{q}\right) \tilde{f}\left(T^{q}\right)^{p^{j} / q} \cdot T^{i} .
$$


By unicity of the expansion we find

$$
\forall i=0, \ldots, p-1: \tilde{\alpha}\left(T^{q}\right) \cdot \tilde{f}_{i}\left(T^{q}\right)=\sum_{j=r+1}^{s} \alpha_{j, i p^{r}}\left(T^{q}\right) \cdot \tilde{f}\left(T^{q}\right)^{p^{j} / q}
$$

so,

$$
\tilde{\alpha} \tilde{f}_{i}=\sum_{j=r+1}^{s} \alpha_{j, i p^{r}} \cdot \tilde{f}^{p^{j} / q} .
$$

Since $\tilde{f}=\Delta^{r+1} f \in A[[X]]^{\text {alg }}$ and $\tilde{\alpha} \neq 0, \tilde{\alpha}, \alpha_{j, i} \in A[T]$ we get that $\tilde{f}_{i}=$ $\Delta^{r} f_{i} \in A[[X]]^{\mathrm{alg}} \Rightarrow f_{i} \in A[[X]]^{\mathrm{alg}}$.

5.3.4. Suppose from now on that we have a valuation ord on a field $K$ with $A$ as its valuation ring and $a$ the maximal ideal. We need not have that $\bigcap_{n=0}^{\infty} a^{n}=0$. But this problem can be solved by remarking that, when $f$ is a-regular, so $f \equiv \varphi+X_{n}^{k} v \bmod X^{\prime}$ with $v$ unit and $\varphi \equiv 0 \bmod a$ we always can find an ideal $\tilde{a} \subset a$ s.t. $\cap \tilde{\alpha}^{n}=0$ and $\varphi \equiv 0 \bmod \tilde{a}$, and if $A$ is complete with the valuation ord, then also in the $\tilde{a}$-topology. Therefore when $A$ is complete, we have a W.P.T. We can extend ord to $A[[X]]$ by letting $\operatorname{ord}\left(\sum a_{i} X^{i}\right)=\inf \left\{\operatorname{ord}\left(a_{i}\right)\right\}$ (Gauss-norm). Recall that $\sum a_{i} X^{i}$ is called separable if the infimum actually is reached. Therefore, it $f$ is separable, we can write it always, after a transformation as

$$
f=\pi \cdot g \quad \text { with ord }(\pi)=\operatorname{ord}(f) .
$$

$\pi \in A$ and $g$ aregular (or shortly, regular) in $X_{n}$. See for instance [Z-S].

We call $A$ separative when every $f \in A[[X]]^{\text {alg }}$ is separable. E.g. rank-one valuation rings.

5.3.5. Lemma. $A$ as above, $A$ separative, $P$ a $D P$ of degree $k$ in $X_{n}$, $P \in A[[X]]^{\mathrm{alg}}$. Suppose $P$ is irreducible in $A[[X]]$; then it is irreducible in $\left(\operatorname{Frac}\left(A\left[\left[X^{\prime}\right]\right]^{\mathrm{alg}}\right)\right)\left[X_{n}\right]$.

Proof. Suppose not, so $P=\alpha \cdot \beta$ where $\alpha, \beta \in\left(\operatorname{Frac} A\left[\left[X^{\prime}\right]\right]^{\text {alg }}\right)\left[X_{n}\right]$ and $\operatorname{deg}_{X_{n}} \alpha, \operatorname{deg}_{X_{n}} \beta<k$. (We will write $\operatorname{deg}$ for $\operatorname{deg}_{X_{n}}$ in the sequel.) So $\exists c \in A\left[\left[X^{\prime}\right]\right]^{\mathrm{alg}}, \tilde{\alpha}, \tilde{\beta} \in A\left[\left[X^{\prime}\right]\right]^{\mathrm{alg}}\left[X_{n}\right]$ s.t.

$$
c P=\tilde{\alpha} \cdot \tilde{\beta}
$$

with $\operatorname{deg} \tilde{\alpha}, \operatorname{deg} \tilde{\beta}<k$. But $A$ is separative, so by (1) of 5.3 .4 we may as well assume that $\tilde{\alpha}, \tilde{\beta}$ are regular in $X_{n}$. By the W.P.T. in $A[[X]]$ we get

$$
P=q \cdot \tilde{\alpha}+\rho
$$

with $\operatorname{deg} \rho<\operatorname{deg} \tilde{\alpha}, q \in A[[X]], \rho \in A\left[\left[X^{\prime}\right]\right]\left[X_{n}\right] ;$ therefore

$$
c P=c q \tilde{\alpha}+c \rho=\tilde{\alpha} \tilde{\beta},
$$


so $\tilde{\alpha} \mid c \rho$ but $\operatorname{deg} \rho<\operatorname{deg} \tilde{\alpha}$ so $\rho=0$, so $P=q \cdot \tilde{\alpha}$ with $\operatorname{deg} \tilde{\alpha}<k$, which contradicts the irreducibility of $P$ in $A[[X]]$.

5.3.6. THEOREM. Let $A$ be a valuation ring, $A$ separative and suppose $A[[X]]$ has a W.P.T.; then $A[[X]]^{\text {alg }}$ also has a W.P.T.

Proof. Take $f \in A[[X]]^{\text {alg }}, f$ regular (= a-regular) in $X_{n}$ of degree $k$. Let $g \in A[[X]]^{\mathrm{alg}}$. By the W.P.T. of $A[[X]]: \exists$ unique $q \in A[[X]], \rho_{i} \in$ $A\left[\left[X^{\prime}\right]\right]$, such that

$$
g=q \cdot f+\sum_{i=0}^{k-1} \rho_{i} X_{n}^{l} .
$$

Since $f$ is regular, we know by the discussion of 5.3.1 that $f$ can be written in the form $f=u \cdot P_{f}$ where $u$ is a unit in $A[[X]]^{\text {alg }}$ and $P_{f}$ is a $D P$ of degree $k$ in $X_{n}, P_{f} \in A[[X]]^{\text {alg }}$. So we can find a "minimal" decomposition

$$
f=\tilde{u} \cdot \prod_{i=1}^{s} P_{i}
$$

with $\tilde{u}$ unit in $A[[X]]^{\text {alg }}$ and $P_{i}$ algebraic DP's in $X_{n}$ such that their degrees are minimal.

Suppose one of the $P_{i}$ is not irreducible in $A[[X]]$, so $P_{i}=\alpha \beta \alpha, \beta$ non-units in $A[[X]]$.

One easily sees that $\alpha, \beta$ need to be regular in $X_{n}$, so can be written as, with $u_{\alpha}, u_{\beta}$ units and $P_{\alpha}, P_{\beta}$ DP's

$$
\alpha=u_{\alpha} P_{\alpha} \text { and } \beta=u_{\beta} P_{\beta}
$$

so $P_{i}=u_{\alpha} u_{\beta} P_{\alpha} P_{\beta}$ is algebraic. From 5.3 .1 we get $u_{\alpha} u_{\beta}, P_{\alpha}$ and $P_{\beta}$ are algebraic, contradicting the minimality of decomposition (2). One checks that, when we can prove (1) for two DP's, then we also have (1) for their product.

So it is sufficient to prove (1) for the irreducible $P_{i}$ 's so we may suppose that $f$ is a DP of degree $k$ in $X_{n}$ and $f$ irreducible in $A[[X]]$, so in $\left(\operatorname{Frac} A\left[\left[X^{\prime}\right]\right]^{\text {alg }}\right)\left[X_{n}\right]$ by 5.3.5.

Let $y_{1}, \ldots, y_{k} \in F$ be the roots of $f$. Suppose first of all that the $y_{l}$ are all distinct. This is certainly the case if $\operatorname{char} A=0$ since $f$ is irreducible over the field Frac $A\left[\left[X^{\prime}\right]\right]^{\text {alg }}$. Substituting in (1) gives us for $j=$ $1, \ldots, k$

$$
g\left(X^{\prime}, y_{j}\right)=\sum_{j=0}^{k-1} \rho_{i} y_{j}^{i}
$$


Consider this as linear system over $F$ with roots $\rho_{i}$ and determinant $\Pi_{j<l}\left(y_{j}-y_{l}\right) \neq 0$ (Vandermonde-type).

So, by Cramer's rule, $\rho_{i}\left(X^{\prime}\right)$ are rational expressions in $y_{j}$ and $g\left(X^{\prime}, y_{j}\right)$ and since the $y_{j}$ are algebraic over $\operatorname{Frac} A[X]$ (for $f$ is) and also $g$ is, one easily verifies that $g\left(X^{\prime}, y_{j}\right)$ are algebraic too, so the $\rho_{i}$ are, and hence also $q \in A[[X]]^{\mathrm{alg}}$.

Suppose now that $\operatorname{char} A=p \neq 0$ and that $f$ has multiple roots. Then there exist an $h \in A\left[\left[X^{\prime}\right]\right]\left[X_{n}\right]$ and $m \in \mathbf{N}$, so that, where $q=p^{m}$, $f=h\left(X_{n}^{q}\right)$ and $h$ is irreducible and has no multiple roots. Since $f \in$ $A[[X]]^{\text {alg }}$, hence $f \in A\left[\left[X^{\prime}\right]\right]^{\text {alg }}\left[X_{n}\right]$ (see 5.3.1), we also get that $h \in$ $A\left[\left[X^{\prime}\right]\right]^{\text {alg }}\left[X_{n}\right]$. Let $s=\operatorname{deg}_{X_{n}} h$, so $\operatorname{deg}_{X_{n}} f=q \cdot s$. Expand $g$ in $q$-base

$$
g=\sum_{i=0}^{q-1} g_{i}\left(X_{n}^{q}\right) X_{n}^{i}
$$

then we know from 5.3.3 that $g_{i}(X) \in A[[X]]^{\text {alg }}$. Apply now the W.P.T. for the DP $h$, which has no multiple roots, so $\exists$ unique $q_{i} \in A[[X]]^{\text {alg }}$ and $\rho_{i} \in A\left[\left[X^{\prime}\right]\right]^{\text {alg }}\left[X_{n}\right], \operatorname{deg}_{X_{n}} \rho_{i} \leq s-1$ s.t. $g_{i}=q_{i} \cdot h+\rho_{i}$. Using expansion (3)

$$
g=\sum_{i=0}^{q-1} g_{i}\left(X_{n}^{q}\right) X_{n}^{i}=\sum_{i=0}^{q-1} q_{l}\left(X_{n}^{q}\right) h\left(X_{n}^{q}\right) X_{n}^{i}+\sum_{i=0}^{q-1} \rho_{i}\left(X_{n}^{q}\right) X_{n}^{i}
$$

Call

$$
\rho=\sum_{i=0}^{q-1} \rho_{i}\left(X_{n}^{q}\right) X_{n}^{i}
$$

$$
\text { with } \operatorname{deg}_{X_{n}} \rho \leq q-1+q(s-1)=q s-1=\operatorname{deg} f-1
$$

hence $g=\left(\sum q_{i}\left(X_{n}^{q}\right) X_{n}^{i}\right) \cdot f+\rho$.

5.4.1. Let's now work in the special type of complete rank-one valuation-rings, defined in 4.2.1 and the PP-system $\stackrel{\circ}{R}_{n}$ of 4.3.1. If $T$ is a finite subset of $\stackrel{R}{R}$, then we denote by

$$
\mathscr{A}_{T}=\{A \in \mathscr{A} \mid T \subset A[[X]]\}
$$

then $\left(\mathscr{A}_{T}, \subset\right.$ (inclusion)) forms a direct system with $\lim _{\mathscr{A}_{T}} A[[X]]=$ $\stackrel{\circ}{R}_{[[X]]}$. One checks that, for $T$ a finite subset of $\stackrel{\circ}{R}$,

$$
\frac{\stackrel{\circ}{R}}{(T) \stackrel{\circ}{R}} \cong \underset{\mathscr{A}_{T}}{\lim } \frac{A[[X]]}{(T) A[[X]]}
$$


5.4.2. TheOREM. The PP-system $R_{[[X]]}=R_{n}$ has a W.P.T.

Proof. Suppose $f$ is regular in $X_{n}$ of degree $k$, so we can write $f$ as

$$
f \equiv \varphi\left(X_{n}\right)+v\left(X_{n}\right) \cdot X_{n}^{k} \bmod \left(X^{\prime}\right)
$$

with $\varphi\left(X_{n}\right) \in \mathcal{O}\left[X_{n}\right], \operatorname{deg} \varphi<k$ and $\operatorname{ord}(\varphi)>0$ and $v\left(X_{n}\right)$ unit in $\mathcal{O}\left[\left[X_{n}\right]\right]$; moreover $v \in R_{[[X]]}$.

Call $E=\left(R_{n} /(f) R_{n}\right)$ where $R_{n}=R_{[[X]]}$. Take $\pi \in \mathcal{O}$, s.t. $\operatorname{ord}(\pi)=$ $\operatorname{ord}(\varphi)>0$. Replacing $A[[X]]$ in 5.2 .1 by $R$, which is complete in the $\pi$-topology, we can prove analogously that $E$ is Hausdorff in the $\pi$-topology. By the remark in 5.3.4. we have a W.P.T. for $\mathcal{O}[[X]]$, which proves the uniqueness in division by $f$.

So we need only to prove that $E$ is finitely-generated as a $R_{n-1}$-module by $1, X_{n}, \ldots, X_{n}^{k-1}$. By the generator-lemma this amounts in proving the same for $E / \pi E$ over $R_{n-1} / \pi R_{n-1}$.

Since $R / \pi \cong \stackrel{\circ}{R} / \pi$, we can find $\tilde{v}, \tilde{f} \in \stackrel{\circ}{R}\left(\tilde{v} \in \mathcal{O}\left[\left[X_{n}\right]\right]\right)$, with $\tilde{v} \equiv v$, $\tilde{f} \equiv f \bmod \pi$ (so $\tilde{v}$ still a unit) and $\tilde{f} \equiv \tilde{v} X_{n}^{k} \bmod \left(\pi, X^{\prime}\right)$.

So

$$
\frac{E}{\pi E}=\frac{R_{n}}{(\pi, f)} \cong \frac{\stackrel{\circ}{R}_{n}}{(\pi, \tilde{f})}=\underset{\mathscr{A}_{\pi, \tilde{f}, \tilde{v}}}{\lim }\left(\frac{A}{\pi A} \frac{[[X]]}{(\tilde{f})}\right)
$$

and

$$
\frac{R_{n-1}}{\pi R_{n-1}} \cong \underset{\mathscr{A}_{\pi, j, \tilde{v}}}{\lim }\left(\frac{A}{\pi A}\right)\left[\left[X^{\prime}\right]\right] .
$$

So, when we can prove that for $A \in \mathscr{A}_{\pi, \tilde{f}, \tilde{v}},(A / \pi A)[[X]] /(\tilde{f})$ is finitely generated as a $A / \pi A\left[\left[X^{\prime}\right]\right]$-module by $1, X_{n}, \ldots, X_{n}^{k}$ we are done. But $(A / \pi A)\left[\left[X^{\prime}\right]\right]$ is complete and Hausdorff with respect to the $\left(X^{\prime}\right)$ topology because $A$ is noetherian and $\left(X^{\prime}\right) \subset \operatorname{rad}(A / \pi A)\left[\left[X^{\prime}\right]\right]$, so $(A / \pi A)\left[\left[X^{\prime}\right]\right]$ is a Zariski-ring. By applying the generator-lemma once again we obtain

$$
\left(\left(\frac{A}{\pi A}\right) \frac{[[X]]}{(\tilde{f})}\right) /\left(X^{\prime}\right) \cong \frac{A}{\pi A} \frac{\left[\left[X_{n}\right]\right]}{\left(\tilde{v} X_{n}^{k}\right)}
$$

with $\tilde{v}$ unit, and this is a finite $(A / \pi A)\left[\left[X^{\prime}\right]\right] /\left(X^{\prime}\right)=A / \pi A$-module generated by $1, \ldots, X_{n}^{k-1}$.

5.5. Proposition. Let $A$ be a ring, a an ideal in $A, A$ complete and Hausdorff in the a-topology. 
Let $\stackrel{\circ}{W}_{n}$ be a PP-system over $A$ and $W_{n}$ the a-adic closure of $\stackrel{\circ}{W}_{n}$ in $A[[X]]$.

$$
\text { If } \stackrel{\circ}{n}_{n} \text { has a W.P.T., then also } W_{n} \text {. }
$$

Proof. Let $f \in W_{n}$ be a-regular in $X_{n}$ of degree $k$, and $g \in W_{n}$. So there exist $f_{m}, g_{m} \in \stackrel{\circ}{W}_{n}$ such that

$$
f_{m} \rightarrow f \text { and } g_{m} \rightarrow g .
$$

Therefore, for $m$ big enough the $f_{m}$ must be a-regular of degree $k$ in $X_{n}$ too. So, by the W.P.T. in $\stackrel{\circ}{W}_{n} \exists$ unique $q_{m} \in \stackrel{\circ}{W}_{n}, \rho_{\text {im }} \in \stackrel{\circ}{W}_{n-1}$

$$
g_{m}=q_{m} \cdot f_{m}+\sum_{i=0}^{k-1} \rho_{i m} X_{n}^{i}
$$

$\forall t: \exists N: f-f_{m}, g_{m}-g_{s} \in a^{t}$ if $m, s>N$, hence in $A / a^{t} A[[X]]$ we get:

$$
q_{m} f+\sum \rho_{i m} X_{n}^{i}=q_{s} f+\sum \rho_{i s} X_{n}^{i} .
$$

But by the W.P.T. in $A / a^{t} A[[X]]$, such an expansion is unique, so

$$
\begin{gathered}
q_{m} \equiv q_{s} \quad \bmod a^{t}, \\
\rho_{i m} \equiv \rho_{i s} \quad \bmod a^{t} ;
\end{gathered}
$$

thus the $\left(q_{m}\right)_{m}$ and the $\left(\rho_{i m}\right)_{m}$ are Cauchy-sequences in $A[[X]]$. Therefore $\exists q \in W_{n}, \rho_{i} \in W_{n-1}: q_{m} \rightarrow q$ and $\rho_{i m} \rightarrow \rho_{i}$ and so

$$
g=q f+\sum_{i=0}^{k-1} \rho_{i} X_{n}^{i} .
$$

The uniqueness of $q$ and $\rho_{i}$ follows from the W.P.T. in $A[[X]]$.

5.5.2. The same proposition holds for $A$ a complete valuation ring, since $A[[X]]$ has a W.P.T. by the remark of 5.3.4.

\section{REFERENCES}

[Ar] M. Artin, Algebraic approximation of structures over complete local rings, Inst. Hautes Etudes, Sci. Publ. Math., 36 (1968), 23-58.

[Ba] G. Bachman, Introduction to p-adic Numbers and Valuation Theory, Academic Press, New York (1964).

[Ch] J. L. Chabert, Anneaux de Fatou, Enseign. Math., (1972), 141-144.

[Chr] G. Christol, Limites uniformes p-adiques de fonctions algébriques, Thèse Sc. Math. Univ. Paris, 6 (1977).

[Co] I. S. Cohen, On the structure and ideal theory of complete local rings, Trans. Amer. Math. Soc., 59 (1946), 54-106.

[C-R] Coste-Roy, Book on semi-algebraic geometry (in preparation). 
[La] S. Lang, Algebra, Addison-Wesley (1965).

[Laf] J. P. Lafon, Séries formelles algébriques, C. R. Acad. Sci. Paris, 20 (1965), 3238-3241.

[L.D.] L. Lipshitz and J. Denef, Algebraic power series and diagonals, J. Number Theory, to appear.

[Ma] H. Matsumura, Commutative Algebra, Benjamin (1970).

[Ra] M. Raynaud, Anneaux Locaux Henséliens, Lect. Notes in Mathematics 169, Springer Verlag (1970).

[Ro] P. Robba, Proprieté d'approximation pour les éléments algèbriques, (Composition Math.)

[Se] J. P. Serre, Corps Locaux, Hermann Paris (1968).

[Z.-S.] O. Zariski and P. Samuel, Commutative Algebra, Van Nostrand Princeton (1967).

Received September 9, 1986.

Ass. Algebra-Topologie

CelestiJnenlaAn 200B

B-3030 LEUVEN, BELGIUM 



\section{PACIFIC JOURNAL OF MATHEMATICS EDITORS}

\author{
V. S. VARADARAJAN \\ (Managing Editor) \\ University of California \\ Los Angeles, CA 90024 \\ HERBERT ClEMENS \\ University of Utah \\ Salt Lake City, UT 84112 \\ R. FINN \\ Stanford University \\ Stanford, CA 94305
}

\author{
HERMANN FLASCHKA \\ University of Arizona \\ Tucson, AZ 85721
}

RAMESh A. GANGolli

University of Washington Seattle, WA 98195

VAUGHAN F. R. JONES

University of California

Berkeley, CA 94720

\author{
ROBION KIRBY \\ University of California \\ Berkeley, CA 94720
}

C. C. MOORE

University of California

Berkeley, CA 94720

HAROLD STARK

University of California, San Diego

La Jolla, CA 92093

\section{ASSOCIATE EDITORS}
R. AREnS
E. F. BECKENBACH
B. H. NEUMANN
F. WOLF
K. YOSHIDA

(1906-1982)

\section{SUPPORTING INSTITUTIONS}

\begin{abstract}
UNIVERSITY OF ARIZONA
UNIVERSITY OF BRITISH COLUMBIA

CALIFORNIA INSTITUTE OF TECHNOLOGY

UNIVERSITY OF CALIFORNIA

MONTANA STATE UNIVERSITY

UNIVERSITY OF NEVADA, RENO

NEW MEXICO STATE UNIVERSITY

OREGON STATE UNIVERSITY
\end{abstract}

\author{
UNIVERSITY OF OREGON \\ UNIVERSITY OF SOUTHERN CALIFORNIA \\ STANFORD UNIVERSITY \\ UNIVERSITY OF HAWAII \\ UNIVERSITY OF TOKYO \\ UNIVERSITY OF UTAH \\ WASHINGTON STATE UNIVERSITY \\ UNIVERSITY OF WASHINGTON
}

The Supporting Institutions listed above contribute to the cost of publication of this Journal, but they are not owners or publishers and have no responsibility for its content or policies.

Mathematical papers intended for publication in the Pacific Journal of Mathematics should be in typed form or offset-reproduced (not dittoed), double spaced with large margins. Please do not use built up fractions in the text of the manuscript. However, you may use them in the displayed equations. Underline Greek letters in red, German in green, and script in blue. The first paragraph must be capable of being used separately as a synopsis of the entire paper. In particular it should contain no bibliographic references. Please propose a heading for the odd numbered pages of less than 35 characters. Manuscripts, in triplicate, may be sent to any one of the editors. Please classify according to the scheme of Math. Reviews, Index to Vol. 39. Supply name and address of author to whom proofs should be sent. All other communications should be addressed to the managing editor, or Elaine Barth, University of California, Los Angeles, California 90024.

There are page-charges associated with articles appearing in the Pacific Journal of Mathematics. These charges are expected to be paid by the author's University, Government Agency or Company. If the author or authors do not have access to such Institutional support these charges are waived. Single authors will receive $\mathbf{5 0}$ free reprints; joint authors will receive a total of $\mathbf{1 0 0}$ free reprints. Additional copies may be obtained at cost in multiples of 50 .

The Pacific Journal of Mathematics is issued monthly as of January 1966. Regular subscription rate: $\$ 190.00$ a year (5 Vols., 10 issues). Special rate: $\$ 95.00$ a year to individual members of supporting institutions.

Subscriptions, orders for numbers issued in the last three calendar years, and changes of address should be sent to Pacific Journal of Mathematics, P.O. Box 969, Carmel Valley, CA 93924, U.S.A. Old back numbers obtainable from Kraus Periodicals Co., Route 100, Millwood, NY 10546.

The Pacific Journal of Mathematics at P.O. Box 969, Carmel Valley, CA 93924 (ISSN 0030-8730) publishes 5 volumes per year. Application to mail at Second-class postage rates is pending at Carmel Valley, California, and additional mailing offices. Postmaster: send address changes to Pacific Journal of Mathematics, P.O. Box 969, Carmel Valley, CA 93924.

PUBLISHED BY PACIFIC JOURNAL OF MATHEMATICS, A NON-PROFIT CORPORATION Copyright (C) 1988 by Pacific Journal of Mathematics 


\section{Pacific Journal of Mathematics}

\section{Vol. 131, No. 2 December, 1988}

Selman Akbulut and Henry Churchill King, Polynomial equations of immersed surfaces .................................... 209

Alberto Baider and Richard C. Churchill, The Campbell-Hausdorff group and a polar decomposition of graded algebra automorphisms ........2 219

Wayne C. Bell and John William Hagood, Separation properties and exact

Radon-Nikodým derivatives for bounded finitely additive measures . . . 237

Dennis J. Garity, James P. Henderson and David G. Wright, Menger

spaces and inverse limits ...............................249

B. Brent Gordon, Algebraically defined subspaces in the cohomology of a

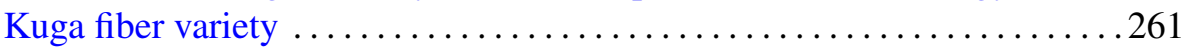

Jeffrey A. Hogan, Weighted norm inequalities for the Fourier transform on connected locally compact groups ........................... 277

Guojun Liao, A study of regularity problem of harmonic maps ..........291

Chin-pi Lu, Modules satisfying ACC on a certain type of colons ......... 303

Kunio Murasugi, Jones polynomials of periodic links

Hans Schoutens, Approximation properties for some non-Noetherian local

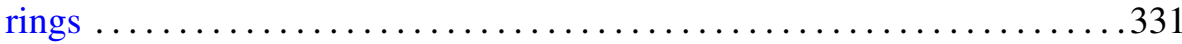

Peter Sjögren, Convergence for the square root of the Poisson kernel ...... 361 Alexandru Ion Suciu, The oriented homotopy type of spun 3-manifolds .393 